\title{
A nodal discontinuous Galerkin finite element method for the poroelastic wave equation
}

\section{Nodal DG for poroelastic system}

\author{
Khemraj Shukla ${ }^{(*)}$ • Jan S Hesthaven • José M. Carcione • Ruichao Ye • \\ Josep de la Puente . Priyank Jaiswal
}

Received: date / Accepted: date

\begin{abstract}
We use the nodal discontinuous Galerkin method with a Lax-Friedrich flux to model the wave propagation in transversely isotropic and poroelastic media. The effect of dissipation due to global fluid flow causes a stiff relaxation term, which is incorporated in the numerical scheme through an operator splitting approach. The well-posedness of the poroelastic system is proved by adopting an approach based on characteristic variables. An error analysis for a plane wave propagating in poroelastic media shows a convergence rate of $O\left(h^{n+1}\right)$. Computational experiments are shown for various combinations of homogeneous and heterogeneous poroelastic media.
\end{abstract}

\section{K. Shukla}

Oklahoma State University, Stillwater, OK.

E-mail: khemraj@okstate.edu

\section{J. S. Hesthaven}

MCSS, EPFL, Switzerland.

E-mail: jan.hesthaven@epfl.ch

\section{J.M.Carcione}

Istituto Nazionale di Oceanografia e di Geofisica Sperimentale (OGS),

Borgo Grotta Gigante 42c,

34010 Sgonico, Trieste, Italy.

E-mail: jcarcione@inogs.it

R.Ye

ExxonMobil, Houston, TX.

E-mail: ruichao.ye@gmail.com

Josep de la Puente

Barcelona Supercomputing Center (BSC), Spain.

E-mail: josep.delapuente@bsc.es

P. Jaiswal

Oklahoma State University, Stillwater, OK.

E-mail: priyank.jaiswal@okstate.edu

(*) Corresponding author.
Keywords Waves · poroelasticity - Lax-Friedrich · attenuation $\cdot$ numerical flux

Mathematics Subject Classification (2010) 35L05. 35S99 - 65M60 - 74J05 - 74J10 - 93C20

\section{Introduction}

The dynamics of fluid-saturated porous media is modeled by the poroelasticity theory, pioneered by Maurice Biot and presented in a series of seminal work during the 1930 s to 1960 s [11]. Porous media acoustics, modeling the propagation of waves in a porous media saturated with a fluid, is an important field of research in various science and engineering disciplines e.g., geophysics, soil mechanics, medical science and civil engineering $[7,1]$. In particular, in the exploration of oil and gas reservoirs, the quantitative estimation of porosity and permeability of rocks is very important to understand the direction of the fluid flow. In general, seismic modeling is performed by approximating the medium as a single phase approximation. These approximations are described by the acoustic $[14,17]$ and elastic $[25$, 30] rheologies and do not account for the loss of energy resulting from the fluid flow. To describe the wave propagation in the porous media, filled with a single phase fluid with an ability to flow through pore networks, Biot proposed the theory of poroelasticity [3-5].

Poroelasticity is a homogenized model of a porous medium, using linear elasticity (Hooke's law) to describe the solid (or skeleton) portion of the medium, linear compressible fluid dynamics to represent the fluid portion, and Darcy's law to model the flow through the pores. Thus, the poroelastic wave equation combines the constitutive relations with the equations of conservation of the momentum and Darcy's law. Global fluid 
flow results into the dissipation of energy due to the relative motion between the solid and fluid particles. Dissipation is incorporated in the equations of motion through a frequency dependent viscodynamic operator $(\psi(t))$. The behavior of the viscodynamic operator depends on the relaxation frequency $\left(\omega_{c}\right)$ [11] of the material. For frequencies lower than the relaxation frequency, $\psi(t)$ is independent of the frequency and compactly supported. In the high frequency range $\left(\geq \omega_{c}\right)$ , $\psi(t)$ becomes frequency dependent and incorporated in the equation of motion through a convolution [9]. Our current work focuses on the poroleasticity in the low-frequency range $\left(<\omega_{c}\right)$. This problem has already been solved by Carcione [9] using the pseudospectral method.

Unlike the acoustic and elastic approximations, wave propagation in a porous medium is a complex phenomenon. In poroelastic materials, three different types of waves appear: 1) A $\mathrm{P}$ wave, similar to an elastic $\mathrm{P}$ wave, with in-phase relative motion between the solid and the fluid; (2) A shear wave, similar to elastic S waves, and (3) A slow P wave or Biot's mode with outof-phase relative motion between the solid and the fluid. Dissipation of energy in the poroelastic system, caused by the relative motion between solid and fluid, causes very low attenuation (and velocity dispersion) in the low-frequency range for $\mathrm{P}$ and $\mathrm{S}$ waves whereas a very strong effect is seen in the slow $\mathrm{P}$ wave $[9,11,8]$. Thus, propagation of the slow $\mathrm{P}$ wave can be seen as a diffusion, which attenuates very rapidly. The slow $\mathrm{P}$ wave propagates at different time scales than those the of $\mathrm{P}$ and $\mathrm{S}$ waves, resulting in a stiff system of equations [8].

A wide variety of numerical methods have been used to solve the system of poroelastic wave equations. A detailed review is presented by Carcione et al. [10]. Most of the methods presented in this paper regard pseudospectral [8,9], staggered pseudospectral [27] and finite-difference methods $[18,15]$ and are based on structured meshes. Santos and Oreña [29] used the finiteelement method to solve the poroelastic wave equation using quadrilateral meshes for spatial discretization. Recent work on the numerical solution of orthotropic poroelasticity is reported by Lemoine et al [24], using a finite-volume method on structured meshes. In our work, we develop a high-order discontinuous Galerkin (DG) methods which is well-suited for simulation of time-domain wave propagation, due to their low dispersion and the ability to accommodate unstructured meshes[19], unlike the finite-difference method.

The time-domain wave propagation, described by a hyperbolic system of partial differential equations, can be solved with explicit time integration if a stability condition is used on the time step length. In general, the finite element method, coupled with explicit time integrator, requires the inversion of a global mass matrix. Spectral element methods avoids the inversion of the global mass matrix for hexahedral elements by choosing the nodal basis function, resulting in a diagonal mass matrix [23]. Inversion of the global mass matrix is avoided in the high order DG method which produces locally invertible matrices. High order DG methods are often used for seismic simulation (elastic approximation) through the use of simplicial meshes $[22,12,32]$.

An inherent challenge in solving the poroelastic system is the treatment of the viscosity-dependent dissipation term. The poroelastic system of equations has the form $\dot{\mathbf{q}}=\mathbf{M q}$, where $\mathbf{q}$ is the wave field vector and $\mathbf{M}$ is a propagation matrix. Since the system is dissipative, the eigenvalues of $\mathbf{M}$ will have a negative real part. The fastest wave in the system will have a small real part whereas the slowest mode (quasi-static) will have a large real part, making the differential equation stiff. The stiffness is more apparent in the lowfrequency regime, whereas in the high frequency regime, separation between the time-scales of the dissipation term and the wave motion is small. The stiffness can be handled in the the poroelastic system by using an implicit scheme for time integration but this will not be a computationally efficient approach. Nevertheless, the viscous term, responsible for the quasi-static mode, is easy to solve analytically, which makes operator splitting a natural choice to handle the stiffness. Carcione and Quiroga-Goode [8] solved the poroacoustic system with operator splitting paired with the pseudospectral method. Operator splitting in a DG method is also explored by de la Puente et al. [13] but to maintain the fast rate of convergence they solved the system of low-frequency poroelastic wave equations (in stressvelocity form) in the diffusive limit by adopting a local space-time DG method [16]. They used a weak form of the numerical scheme, which imposes smoothness on the test functions and is very well suited for the non-linear conservation law to resolve shocks and subsonic flow. In another study by Ward et al. [31] the system of poroelastic wave equations, expressed in the strain-velocity formulation, is solved by using the upwind flux in an isotropic acoustic-poroelastic combination. In DG methods, the flux is applied at the shared edges of elements to recover the global solution. The application of upwind flux causes less dissipation but is more computer intensive as it requires an eigenvalue decomposition of Jacobian matrices. The eigenvalues and eigenvectors of the Jacobian, corresponding to a generic poroelastic medium is not trivial and poses a computational challenge. Furthermore, the upwind flux for an anisotropic medium requires the rotation of eigenvec- 
tors along normals of each edges of elements, although this can be avoided by using the Lax-Friedrich flux.

In this work, we have used a Lax-Friedrich flux [20] which requires knowledge of the maximum speed present in the system to stabilize the numerical scheme. We used a plane-wave approach to compute the maximum speed. Unlike an upwind flux, Lax-Friedrich flux is very generic and can be extended from an isotropic to an anisotropic medium. We have used the $4^{\text {th }}$-order accurate low storage explicit Runge-Kutta scheme for time integration of the non-dissipative (i.e non-stiff part) part of the system. The novelties of our approach are i) We use a coupled first-order low-frequency poroelastic wave equation in conservation form for a transversely anisotropic media. ii) We prove well-posedness of the poroelastic system. In the usual sense, well-posedness of the system admits a unique solution of the system bounded in $L^{2}$ of the boundary or forcing data. iii) We derive a self-consistent DG strong formulation with a Lax-Friedrich flux. iv) We verify the method by comparing the analytical and numerical solutions. v) We perform various computational experiments to study the slow $\mathrm{P}$ wave in isotropic and anisotropic media.

\section{System of equations describing poroelastic wave equation in transversely isotropic medium}

In this section, we discuss Biot's equations of poroelasticity but readers are advised to refer to Biot's original papers [3-5] and [11] for further detail.

\subsection{Stress-strain relations}

The constitutive equations for an inhomogeneous and transversely isotropic poroelastic media is expressed as $[9,2]$

$$
\begin{aligned}
& \partial_{t} \tau_{x x}=c_{11}^{u} \partial_{x} v_{x}+c_{13}^{u} \partial_{z} v_{z}+\alpha_{1} M\left(\partial_{x} q_{x}+\partial_{z} q_{z}\right)+\partial_{t} s_{11} \\
& \partial_{t} \tau_{z z}=c_{13}^{u} \partial_{x} v_{x}+c_{33}^{u} \partial_{z} v_{z}+\alpha_{3} M\left(\partial_{x} q_{x}+\partial_{z} q_{z}\right)+\partial_{t} s_{33}
\end{aligned}
$$

$\partial_{t} \tau_{x z}=c_{55}^{u}\left(\partial_{z} v_{x}+\partial_{x} v_{z}\right)+\partial_{t} s_{55}$

$$
\partial_{t} p=-\alpha_{1} M \partial_{x} v_{x}-\alpha_{3} M \partial_{z} v_{z}-M\left(\partial_{x} q_{x}+\partial_{z} q_{z}\right)+\partial_{t} s_{f}, \text { as [9] }
$$

where $\tau_{x x}, \tau_{z z}$ and $\tau_{x z}$ are the total stresses, $p$ is fluid pressure, the $v^{\prime} s$ and $q^{\prime} s$ are the solid and fluid (relative to solid) particle velocities, respectively, $c_{i j}^{u}, i, j=$ $1, \ldots, 6$ are the undrained components of the elastic stiffness tensor, $M$ is an elastic modulus and $\alpha_{k}, k=1,3$ are Biot's effective coefficients. $s_{i j}$ and $s_{f}$ are the solid and fluid forcing functions, respectively. The conventions are that $\partial_{t}, \partial_{x}$ and $\partial_{z}$ denote time derivative and spatial derivative operator in $x$ and $z$ directions, respectively. The basic underlying assumption in estimating the coefficients is that anisotropy of the porous solid frame is caused by the directional arrangement of the grains. The undrained coefficients $c_{i j}^{u}$ are expressed in terms of drained coefficients, $c_{i j}$, as

$c_{11}^{u}=c_{11}+\alpha_{1}^{2} M$

$c_{33}^{u}=c_{33}+\alpha_{3}^{2} M$,

$c_{13}^{u}=c_{13}+\alpha_{1} \alpha_{3} M$,

$c_{55}^{u}=c_{55}$.

Effective coefficients $\alpha$ and modulus $M$ are given by [11]

$\alpha_{1}=1-\frac{c_{11}+c_{12}+c_{13}}{3 K_{s}}$,
$\alpha_{3}=1-\frac{2 c_{13}+c_{33}}{3 K_{s}}$,
$M=\frac{K_{s}^{2}}{D-\left(2 c_{11}+c_{33}+2 c_{12}+4 c_{13}\right)}$,

where $K_{s}$ is the bulk modulus of the grains and

$D=K s\left(1-\phi+\phi K_{s} K_{f}^{-1}\right)$,

with $K_{f}$ being the fluid bulk modulus and $\phi$ the porosity.

\subsection{Dynamical equations and Darcy's law}

The dynamic equations describing the wave propagation in a transversely isotropic heterogeneous porous medium, are given by $[5,11]$

$\partial_{x} \tau_{x x}+\partial_{z} \tau_{x z}=\rho \partial_{t} v_{x}+\rho_{f} \partial_{t} q_{x}$

$\partial_{x} \tau_{x z}+\partial_{z} \tau_{z z}=\rho \partial_{t} v_{z}+\rho_{f} \partial_{t} q_{z}$

where $\rho=(1-\phi) \rho_{s}+\phi \rho_{f}$ is the bulk density, and $\rho_{s}$ and $\rho_{f}$ are the solid and fluid density, respectively.

The generalized dynamic Darcy's law, governing the fluid flow in an anisotropic porous media, is expressed

$-\partial_{x} p=\rho_{f} \partial_{t} v_{x}+\psi_{1} * \partial_{t} q_{x}$,

$-\partial_{z} p=\rho_{f} \partial_{t} v_{z}+\psi_{3} * \partial_{t} q_{z}$,

where "*" denotes the time convolution operators and $\psi_{i}, i=1,3$ are the time-dependent Biot's viscodynamic operator in the $x$ and $z$ directions. In the low frequency 
range, i.e., for frequencies lower than $\omega_{c}=\min \left(\frac{\eta \phi}{\rho_{f} T_{i} \kappa_{i}}\right)$, $\psi_{i}$ can be expressed as

$\psi_{i}(t)=m_{i} \delta(t)+\left(\eta / \kappa_{i}\right) H(t)$

where $m_{i}=T_{i} \rho_{f} / \phi$, with $T_{i}$ being the tortuosity, $\eta$ the fluid viscosity, and $\kappa_{1}$ and $\kappa_{3}$ the principal components of the global permeability tensor, while $\delta(t)$ is Dirac's function and $H(t)$ the Heaviside step function. Substituting (17) in (15) and (16), we get

$-\partial_{x} p=\rho_{f} \partial_{t} v_{x}+m_{1} \partial_{t} q_{x}+\frac{\eta}{\kappa_{1}} q_{x}$

$-\partial_{z} p=\rho_{f} \partial_{t} v_{z}+m_{3} \partial_{t} q_{z}+\frac{\eta}{\kappa_{1}} q_{z}$.

Equations (13), (14),(18) and (19) yield

$\partial_{t} v_{x}=\beta_{11}^{(1)}\left(\partial_{x} \tau_{x x}+\partial_{z} \tau_{x z}\right)-\beta_{12}^{(1)}\left(\partial_{x} p+\frac{\eta}{\kappa_{1}} q_{x}\right)$

$\partial_{t} v_{z}=\beta_{11}^{(3)}\left(\partial_{x} \tau_{x z}+\partial_{z} \tau_{z z}\right)-\beta_{12}^{(3)}\left(\partial_{z} p+\frac{\eta}{\kappa_{3}} q_{z}\right)$

$\partial_{t} q_{x}=\beta_{21}^{(1)}\left(\partial_{x} \tau_{x x}+\partial_{z} \tau_{x z}\right)-\beta_{22}^{(1)}\left(\partial_{x} p+\frac{\eta}{\kappa_{1}} q_{x}\right)$

$\partial_{t} q_{z}=\beta_{21}^{(3)}\left(\partial_{x} \tau_{x z}+\partial_{z} \tau_{z z}\right)-\beta_{22}^{(3)}\left(\partial_{z} p+\frac{\eta}{\kappa_{3}} q_{z}\right)$,

where

$\left[\begin{array}{cc}\beta_{11}^{(k)} & \beta_{12}^{(k)} \\ \beta_{21}^{(k)} & \beta_{22}^{(k)}\end{array}\right]=\left(\rho_{f}^{2}-\rho m_{k}\right)^{-1}\left[\begin{array}{cc}-m_{k} & \rho_{f} \\ \rho_{f} & -\rho\end{array}\right]$.

\subsection{Equations in a system form}

To simplify the notation, we introduce a system form of the equations by combining equations (1)-(4) and (20)(23). The conservation form of the system of poroelastic wave equations is

$\partial_{t} \mathbf{q}+\nabla \cdot\left(\mathcal{A}_{n} \mathbf{q}\right)=\mathbf{D q}+\mathbf{f}(\mathbf{t})$

where $\mathbf{q}=\left[\begin{array}{llllllll}p & \tau_{x x} & \tau_{z z} & \tau_{x z} & v_{x} & v_{z} & q_{x} & q_{z}\end{array}\right]^{T}, \mathcal{A}_{n}=n_{1} A_{1}+$ $n_{2} A_{2}$, and $\mathbf{n}=\left[\begin{array}{ll}n_{1} & n_{2}\end{array}\right]^{T}$ denotes the outward interface unit normal vector. Furthermore

$$
\begin{aligned}
\mathbf{A}_{1}= & {\left[\begin{array}{cccccccc}
0 & 0 & 0 & 0 & \alpha_{1} M & 0 & M & 0 \\
0 & 0 & 0 & 0 & -c_{11}^{u} & 0 & -\alpha_{1} M & 0 \\
0 & 0 & 0 & 0 & -c_{13}^{u} & 0 & -\alpha_{3} M & 0 \\
0 & 0 & 0 & 0 & 0 & c_{55}^{u} & 0 & 0 \\
\beta_{12}^{(1)} & -\beta_{11}^{(1)} & 0 & 0 & 0 & 0 & 0 & 0 \\
0 & 0 & 0 & -\beta_{11}^{(3)} & 0 & 0 & 0 & 0 \\
\beta_{22}^{(1)} & -\beta_{21}^{(1)} & 0 & 0 & 0 & 0 & 0 & 0 \\
0 & 0 & 0 & -\beta_{21}^{(3)} & 0 & 0 & 0 & 0
\end{array}\right], } \\
\mathbf{A}_{2}= & {\left[\begin{array}{ccccccccc}
0 & 0 & 0 & 0 & 0 & \alpha_{3} M & 0 & M \\
0 & 0 & 0 & 0 & 0 & -c_{13}^{u} & 0 & -\alpha_{1} M \\
0 & 0 & 0 & 0 & 0 & -c_{33}^{u} & 0 & -\alpha_{3} M \\
0 & 0 & 0 & 0 & -c_{55}^{u} & 0 & 0 & 0 \\
0 & 0 & 0 & -\beta_{11}^{(1)} & 0 & 0 & 0 & 0 \\
\beta_{12}^{(3)} & 0 & -\beta_{11}^{(3)} & 0 & 0 & 0 & 0 & 0 \\
0 & 0 & 0 & -\beta_{21}^{(1)} & 0 & 0 & 0 & 0 \\
\beta_{22}^{3} & 0 & -\beta_{21}^{(3)} & 0 & 0 & 0 & 0 & 0
\end{array}\right], }
\end{aligned}
$$

$$
\mathbf{D}=\left[\begin{array}{cccccccc}
0 & 0 & 0 & 0 & 0 & 0 & 0 & 0 \\
0 & 0 & 0 & 0 & 0 & 0 & 0 & 0 \\
0 & 0 & 0 & 0 & 0 & 0 & 0 & 0 \\
0 & 0 & 0 & 0 & 0 & 0 & 0 & 0 \\
0 & 0 & 0 & 0 & 0 & 0 & \frac{-\beta_{12}^{(1)} \eta}{\kappa_{1}} \\
0 & 0 & 0 & 0 & 0 & 0 & 0 & \frac{-\beta_{12}^{(3)} \eta}{\kappa_{3}} \\
0 & 0 & 0 & 0 & 0 & 0 & \frac{-\beta_{22}^{(1)} \eta}{\kappa_{1}} & 0 \\
0 & 0 & 0 & 0 & 0 & 0 & 0 & \frac{-\beta_{22}^{(3)} \eta}{\kappa_{3}}
\end{array}\right],
$$

and $f(t)=\left[\begin{array}{llll}\partial_{t} s_{11} & \partial_{t} s_{33} & \partial_{t} s_{55} & \partial_{t} s_{f}\end{array}\right]^{T}$.

\section{Well-posedness of the poroelastic system of equations}

In the velocity-stress formulation, the governing equations describing wave propagation in a region $\Omega$ enclosed by its boundary $\partial \Omega$ and filled with heterogeneous transversely isotropic porous media, is expressed as

$$
\begin{aligned}
\mathbf{q}_{t}+\partial_{x}\left(\mathbf{A}_{\mathbf{1}} \mathbf{q}\right)+\partial_{y}\left(\mathbf{A}_{\mathbf{2}} \mathbf{q}\right) & =\mathbf{D} \mathbf{q}+\mathbf{f}(\mathbf{t}) \\
\mathbf{x} & =(x, y) \in \Omega, t>0, \\
\mathbf{q} & =\mathbf{h}(\mathbf{x}), \quad \mathbf{x} \in \Omega, t=0, \\
\mathcal{B} \mathbf{q} & =\mathbf{g}(\mathbf{x}, t), \quad \mathbf{x} \in \partial \Omega, t \geq 0 .
\end{aligned}
$$

To prove the well-posedness of the system (29), first, we seek a symmetrizer for $\mathbf{A}_{\mathbf{1}}$ and $\mathbf{A}_{\mathbf{2}}$. 
The strain or potential energy of the poroelastic system is [11],

$E_{s}=\frac{1}{2} \boldsymbol{\tau}^{T} \mathbf{C} \boldsymbol{\tau}$

where $\boldsymbol{\tau}=\left[\begin{array}{llll}\tau_{x x} & \tau_{z z} & \tau_{x z} & p\end{array}\right]^{T}$ and $\mathbf{C}$ is a symmetric undrained compliance matrix,

$\mathbf{C}=\left[\begin{array}{cccc}C_{11} & C_{12} & 0 & C_{14} \\ C_{12} & C_{22} & 0 & C_{24} \\ 0 & 0 & C_{33} & 0 \\ C_{14} & C_{24} & 0 & C_{44}\end{array}\right]$,

where $C_{11}=\frac{c_{33}}{c_{11} c_{33}-c_{13}^{2}}, C_{12}=-\frac{c_{13}}{c_{11} c_{33}-c_{13}^{2}}$,

$C_{14}=\frac{\alpha_{1} c_{33}-\alpha_{3} c_{13}}{c_{11} c_{33}-c_{13}^{2}}, C_{22}=\frac{c_{11}}{c_{11} c_{33}-c_{13}^{2}}$,

$C_{24}=\frac{\alpha_{3} c_{11}-\alpha_{1} c_{13}}{c_{11} c_{33}-c_{13}^{2}}, C_{33}=\frac{1}{c_{55}}$,

and $C_{44}=\frac{\alpha_{3} c_{11}-\alpha_{1} c_{13}}{c_{11} c_{33}-c_{13}^{2}}$.

The kinetic energy of the poroelastic system can be written as [11]

$E_{v}=\frac{1}{2}\left[\rho \mathbf{v}^{T} \mathbf{v}+2 \rho_{f} \mathbf{q}^{T} \mathbf{v}+\mathbf{q}_{\mathbf{f}}^{T} \mathbf{m} \mathbf{q}_{\mathbf{f}}\right]$,

where $\mathbf{v}=\left[\begin{array}{ll}v_{x} & v_{z}\end{array}\right]^{T}, \mathbf{q}_{\mathbf{f}}=\left[\begin{array}{ll}q_{x} & q_{z}\end{array}\right]^{T}$ and $\mathbf{m}=\left(\begin{array}{cc}m_{1} & 0 \\ 0 & m_{3}\end{array}\right)$. To construct a simultaneous symmetrizer $(\mathcal{H}$, a symmetric positive definite operator) for Jacobians $\mathbf{A}_{\mathbf{1}}$ and $\mathbf{A}_{\mathbf{2}}$, a block-diagonal matrix with non-zero elements, being the Hessian of (30) and (32), is expressed as

$\mathcal{H}=\left[\begin{array}{cc}\mathbf{E}_{11} & 0 \\ 0 & \mathbf{E}_{22}\end{array}\right]$

where $E_{11}=\operatorname{Hessian}\left(E_{s}\right)=\Delta E_{s}\left(\begin{array}{llll}p & \tau_{x x} & \tau_{z z} & \tau_{x z}\end{array}\right)$ and $E_{22}=\operatorname{Hessian}\left(E_{v}\right)=\Delta E_{s}\left(\begin{array}{llll}v_{x} & v_{z} & q_{x} & q_{z}\end{array}\right)$. Hence $\mathbf{E}_{11}$.

$\mathbf{E}_{11}=\left[\begin{array}{cccc}e_{11} & e_{12} & e_{13} & 0 \\ e_{21} & e_{22} & e_{23} & 0 \\ e_{13} & e_{23} & e_{33} & 0 \\ 0 & 0 & 0 & e_{44}\end{array}\right]$

and

$\mathbf{E}_{22}=\left[\begin{array}{cccc}\rho & 0 & \rho_{f} & 0 \\ 0 & \rho & 0 & \rho_{f} \\ \rho_{f} & 0 & m_{1} & 0 \\ 0 & \rho_{f} & 0 & m_{3}\end{array}\right]$, where $e_{11}=\frac{\alpha_{1}^{3} c_{33}+\alpha_{3}^{2} c_{11}-2 \alpha_{1} \alpha_{3} c_{13}}{c_{11} c_{33}-c_{13}^{2}}+\frac{1}{M}, e_{12}=$ $\frac{\alpha_{1} c_{33}-\alpha_{3} c_{13}}{c_{11} c_{33}-c_{13}^{2}}, e_{13}=\frac{\alpha_{3} c_{11}-\alpha_{1} c_{13}}{c_{11} c_{33}-c_{13}^{2}}, e_{22}=\frac{c_{33}}{c_{11} c_{33}-c_{13}^{2}}$, $e_{23}=-\frac{c_{13}}{c_{11} c_{33}-c_{13}^{2}}, e_{33}=-\frac{c_{13}}{c_{11} c_{33}-c_{13}^{2}}$ and $e_{44}=$ $\frac{1}{c_{55}}$.

Applying $\mathcal{H}$ to $\mathbf{A}_{\mathbf{1}}, \mathbf{A}_{\mathbf{2}}$ and $\mathbf{D}$ yields

$\mathcal{H} \mathbf{A}_{1}=\widetilde{\mathbf{A}}_{1}=\left[\begin{array}{cccccccc}0 & 0 & 0 & 0 & 0 & 0 & 1 & 0 \\ 0 & 0 & 0 & 0 & -1 & 0 & 0 & 0 \\ 0 & 0 & 0 & 0 & 0 & 0 & 0 & 0 \\ 0 & 0 & 0 & 0 & 0 & -1 & 0 & 0 \\ 0 & -1 & 0 & 0 & 0 & 0 & 0 & 0 \\ 0 & 0 & 0 & -1 & 0 & 0 & 0 & 0 \\ 1 & 0 & 0 & 0 & 0 & 0 & 0 & 0 \\ 0 & 0 & 0 & 0 & 0 & 0 & 0 & 0\end{array}\right]$,

$$
\begin{aligned}
& \mathcal{H} \mathbf{A}_{2}=\widetilde{\mathbf{A}}_{2}= {\left[\begin{array}{cccccccc}
0 & 0 & 0 & 0 & 0 & 0 & 0 & 1 \\
0 & 0 & 0 & 0 & 0 & 0 & 0 & 0 \\
0 & 0 & 0 & 0 & 0 & -1 & 0 & 0 \\
0 & 0 & 0 & 0 & -1 & 0 & 0 & 0 \\
0 & 0 & 0 & -1 & 0 & 0 & 0 & 0 \\
0 & 0 & -1 & 0 & 0 & 0 & 0 & 0 \\
0 & 0 & 0 & 0 & 0 & 0 & 0 & 0 \\
1 & 0 & 0 & 0 & 0 & 0 & 0 & 0
\end{array}\right], } \\
& \mathcal{H} \mathbf{D}=\widetilde{\mathbf{D}}=\left[\begin{array}{cccccccc}
0 & 0 & 0 & 0 & 0 & 0 & 0 & 0 \\
0 & 0 & 0 & 0 & 0 & 0 & 0 & 0 \\
0 & 0 & 0 & 0 & 0 & 0 & 0 & 0 \\
0 & 0 & 0 & 0 & 0 & 0 & 0 & 0 \\
0 & 0 & 0 & 0 & 0 & 0 & 0 & 0 \\
0 & 0 & 0 & 0 & 0 & 0 & 0 & 0 \\
0 & 0 & 0 & 0 & 0 & 0 & -\frac{\eta}{\kappa_{1}} & 0 \\
0 & 0 & 0 & 0 & 0 & 0 & 0 & -\frac{\eta}{\kappa_{3}}
\end{array}\right],
\end{aligned}
$$

where $\widetilde{\mathbf{A}}_{1}$ and $\widetilde{\mathbf{A}}_{2}$ are symmetric and $\widetilde{\mathbf{D}}$ is a negative definite matrix. $\mathcal{H}$ is positive-definite and symmetrizes $\mathbf{A}_{1}$ and $\mathbf{A}_{2}$. Thus well-posedness of (29) follows by energy estimate $E(t)$, which is expressed as

$E(t)=\frac{1}{2} \mathbf{q}^{T} \mathcal{H} \mathbf{q}$

It suffices to consider (29) for $\eta=0$ and $\mathbf{f}=\mathbf{0}$. Multiplying (29) with $\mathbf{q}^{\mathbf{T}} \mathcal{H}$, integrating over $\Omega$ and using the divergence theorem, we recover,

$\frac{d E(t)}{d t}=\oint_{\partial \Omega} \mathbf{q}^{\mathbf{T}} \mathbf{A}(\mathbf{n}) \mathbf{q} d \mathbf{x}$, 
where $\oint_{\partial \Omega}$ denotes the surface integral over $\partial \Omega$ and

$\mathbf{A}(\mathbf{n})=\sum_{i=1}^{2} n_{i} \widetilde{\mathbf{A}}_{i}=\left[\begin{array}{cccccccc}0 & 0 & 0 & 0 & 0 & 0 & n_{1} & n_{2} \\ 0 & 0 & 0 & 0 & -n_{1} & 0 & 0 & 0 \\ 0 & 0 & 0 & 0 & 0 & -n_{2} & 0 & 0 \\ 0 & 0 & 0 & 0 & -n_{2} & -n_{1} & 0 & 0 \\ 0 & -n_{1} & 0 & -n_{2} & 0 & 0 & 0 & 0 \\ 0 & 0 & -n_{2} & -n_{1} & 0 & 0 & 0 & 0 \\ n_{1} & 0 & 0 & 0 & 0 & 0 & 0 & 0 \\ n_{2} & 0 & 0 & 0 & 0 & 0 & 0 & 0\end{array}\right]$.

Since $\mathbf{A}(\mathbf{n})$ is symmetric, there exists an unitary matrix $\mathbf{S}(\mathbf{n})$ such that $\mathbf{A}(\mathbf{n})=\mathbf{S}(\mathbf{n})^{\mathbf{T}} \boldsymbol{\Lambda} \mathbf{S}(\mathbf{n})$. The expression for $\mathbf{S}$ and $\Lambda$ are given as

$$
\mathbf{S}(\mathbf{n})=\left[\begin{array}{cccccccc}
0 & 0 & -\frac{1}{n_{2}} & \frac{1}{n_{2}} & 0 & 0 & 0 & 0 \\
0 & -\frac{n_{2}}{n_{1}} & 0 & 0 & -\frac{n_{1}}{\alpha_{-}} & \frac{n_{1}}{\alpha_{\bar{n}_{2}}} & \frac{n_{1}}{\alpha_{+}} & -\frac{n_{1}}{\alpha_{+}} \\
0 & -\frac{n_{1}}{n_{2}} & 0 & 0 & \frac{n_{2}}{\alpha_{-}} & -\frac{n_{2}}{\alpha_{-}} & \frac{\frac{n_{2}}{\alpha_{+}}}{n_{+}} & -\frac{n_{2}}{\alpha_{+}} \\
0 & 1 & 0 & 0 & -\frac{-n_{1}+n_{2}}{\alpha_{-}} & -\frac{n_{1}-n_{2}}{\alpha_{-}} & \frac{-n_{1}-n_{2}}{\alpha_{+}} & -\frac{n_{1}+n_{2}}{\alpha_{+}} \\
0 & 0 & 0 & 0 & -1 & -1 & 1 & 1 \\
0 & 0 & 0 & 0 & 1 & 1 & 1 & 1 \\
-\frac{n_{2}}{n_{1}} & 0 & \frac{n_{1}}{n_{2}} & \frac{n_{1}}{n_{2}} & 0 & 0 & 0 & 0 \\
1 & 0 & 1 & 1 & 0 & 0 & 0 & 0
\end{array}\right],
$$

where $\boldsymbol{\Lambda}=\operatorname{diag}\left[\begin{array}{llllllll}\lambda_{1} & \lambda_{2} & \lambda_{3} & \lambda_{4} & \lambda_{5} & \lambda_{6} & \lambda_{7} & \lambda_{8}\end{array}\right]=$

$\operatorname{diag}\left[00-11-\alpha_{-} \alpha_{-}-\alpha_{+} \alpha_{+}\right]$with

$$
\alpha_{ \pm}=\sqrt{1 \pm n_{1} n_{2}} \text {. }
$$

The characteristic state vector $\mathbf{R}$ is expressed as

$\mathbf{R}(\mathbf{n})=\mathbf{S}^{\mathbf{T}}(\mathbf{n}) \mathbf{q}=\left[R_{1}, R_{2}, R_{3}, R_{4}, R_{5}, R_{6}, R_{7}, R_{8}\right]^{T}$

Thus we have

$\mathbf{q}^{T} \mathbf{A}(\mathbf{n}) \mathbf{q}=\mathbf{R}^{T} \Lambda(n) \mathbf{R}(\mathbf{n})=\sum_{i=1}^{8}\left(\lambda_{i} R_{i}^{2}\right)$

Substituting (40) in (38), we recover

$\frac{d E(t)}{d t}=\sum_{i=1}^{8} I S_{i}, \quad I S_{i}=\oint_{\partial \Omega}\left(\lambda_{i} R_{i}^{2}\right) d \mathbf{x}$.

Equation (41) implies that the net rate of change of the energy with respect to time is estimated by summing the surface integrals. Surface integrals for $i=4,6,8$ contribute to the energy of the system from the boundary. Surface integrals for $i=3,5,7$ extract out the energy from the system, if the integral is not zero. Thus $R_{4}, R_{6}, R_{8}$ are incoming characteristics and $R_{3}, R_{5}, R_{7}$ are outgoing characteristics. For $\lambda_{1}=\lambda_{2}=0$ there is no addition or subtraction of energy to the system thus, characteristic variables $R_{1}, R_{2}$ are stationary characteristics. Since $\lambda_{3}, \lambda_{5}$, and $\lambda_{7}$ are negative,

$\frac{d E(t)}{d t} \leq \oint_{\partial \Omega}\left(R_{4}^{2}+\alpha_{-} R_{6}^{2}+\alpha_{+} R_{8}^{2}\right) d \mathbf{x}$, which implies that if a boundary condition is of the form

$\mathcal{B} \mathbf{q}=\left[\begin{array}{l}\mathbf{s}_{4}^{T} \\ \mathbf{s}_{6}^{T} \\ \mathbf{s}_{8}^{T}\end{array}\right] \mathbf{q}=\mathbf{g}(\mathbf{x}, t)=\left[\begin{array}{l}\mathbf{g}_{1}(\mathbf{x}, t) \\ \mathbf{g}_{2}(\mathbf{x}, t) \\ \mathbf{g}_{3}(\mathbf{x}, t)\end{array}\right]$ and $\mathbf{x} \in \partial \Omega$

and we have

$$
\begin{aligned}
\oint_{\partial \Omega}\left(R_{4}^{2}+\alpha_{-} R_{6}^{2}+\alpha_{+} R_{8}^{2}\right) & \leq \max \left(1, \alpha_{-}, \alpha_{+}\right)\left(\oint_{\partial \Omega} \mathbf{q}^{T} \mathbf{s}_{\mathbf{4}} \mathbf{s}_{\mathbf{4}}{ }^{T} \mathbf{q} d \mathbf{x}\right. \\
& \left.+\oint_{\partial \Omega} \mathbf{q}^{T} \mathbf{s}_{\mathbf{6}} \mathbf{s}_{\mathbf{6}}{ }^{T} \mathbf{q} d \mathbf{x}+\oint_{\partial \Omega} \mathbf{q}^{T} \mathbf{s}_{\mathbf{8}} \mathbf{s}_{\mathbf{8}}{ }^{T} \mathbf{q} d \mathbf{x}\right) \\
& =\alpha \oint_{\partial \Omega}|\mathbf{g}(\mathbf{x}, t)|^{2} d \mathbf{x}=\alpha G(t),
\end{aligned}
$$

with $\alpha=\max \left(1, \alpha_{-}, \alpha_{+}\right)$.

Thus we recover

$\frac{d E(t)}{d t} \leq G(t)$

Integrating w.r.t. time yields

$E(t) \leq \int_{0}^{t} G(\chi) d \chi+E(0) \leq E(0)+t \max _{\chi \in[0, t]} G(\chi)$

Alternatively,

$\int_{\Omega} \mathbf{q}^{\mathbf{T}}(\mathbf{x}, t) \mathbf{H} \mathbf{q}(\mathbf{x}, t) d \mathbf{x} \leq \int_{\partial \Omega} \mathbf{h}^{T}(\mathbf{x}) \mathbf{H h}(\mathbf{x}) d \mathbf{x}+t\left(\max _{\chi \in[0, t]} G(\chi)\right)$.

Equation (44) states that the energy of the system is bounded by the initial condition $\mathbf{h}(\mathbf{x}, t)$ and the boundary condition $\mathbf{g}(\mathbf{x}, t)$, prescribed by (29). Thus, the poroelastic system of equation, defined by equation (29), is a well-posed problem. This result is summarized in the theorem.

Theorem 1 Assume there exist a smooth solution to (29). If the boundary condition is given of the form

$\mathcal{B} \mathbf{q}=\left[\begin{array}{l}\mathbf{s}_{4}^{T} \\ \mathbf{s}_{6}^{T} \\ \mathbf{s}_{8}^{T}\end{array}\right] \mathbf{q}=\mathbf{g}(\mathbf{x}, t)=\left[\begin{array}{l}\mathbf{g}_{1}(\mathbf{x}, t) \\ \mathbf{g}_{2}(\mathbf{x}, t) \\ \mathbf{g}_{3}(\mathbf{x}, t)\end{array}\right]$ and $\mathbf{x} \in \partial \Omega$,

then the IBVP (29) is well-posed and $\mathbf{q}$ satisfies the estimate

$\int_{\Omega} \mathbf{q}^{\mathbf{T}}(t) \mathbf{H} \mathbf{q}(t) d \mathbf{x} \leq \int \mathbf{h}^{T}(\mathbf{x}) \mathbf{H h}(\mathbf{x}) d \mathbf{x}+t \max _{\chi \in[0, t]} G(\chi)$,

with

$G(t)=\alpha \oint_{\partial \Omega}|\mathbf{g}(\mathbf{x}, \mathbf{t})|^{2} d \mathbf{x}, \quad \alpha=\max _{\mathbf{x} \in \partial \Omega}\left(1, \alpha_{+}, \alpha_{-}\right)$. 
Table 1 Material properties for sandstone saturated with brine $[8]$

\begin{tabular}{cc}
\hline Properties & Sandstone (Isotropic) \\
\hline$K_{s}(\mathrm{GPa})$ & 40 \\
$\rho_{s}\left(\mathrm{~kg} / \mathrm{m}^{3}\right)$ & 2500 \\
$\phi$ & 0.2 \\
$\kappa\left(10^{-15} \mathrm{~m}^{2}\right)$ & 600 \\
$K_{f}(\mathrm{GPa})$ & 2.5 \\
$\rho_{f}\left(\mathrm{~kg} / \mathrm{m}^{3}\right)$ & 1040 \\
$T_{1}$ & 3 \\
$T_{3}$ & 3 \\
$\eta\left(10^{-3} \mathrm{Kg} / \mathrm{m} . \mathrm{s}\right)$ & 1 \\
$\lambda^{*}(\mathrm{~m} / \mathrm{s})$ & 3800
\end{tabular}

${ }^{*}$ computed in this study.

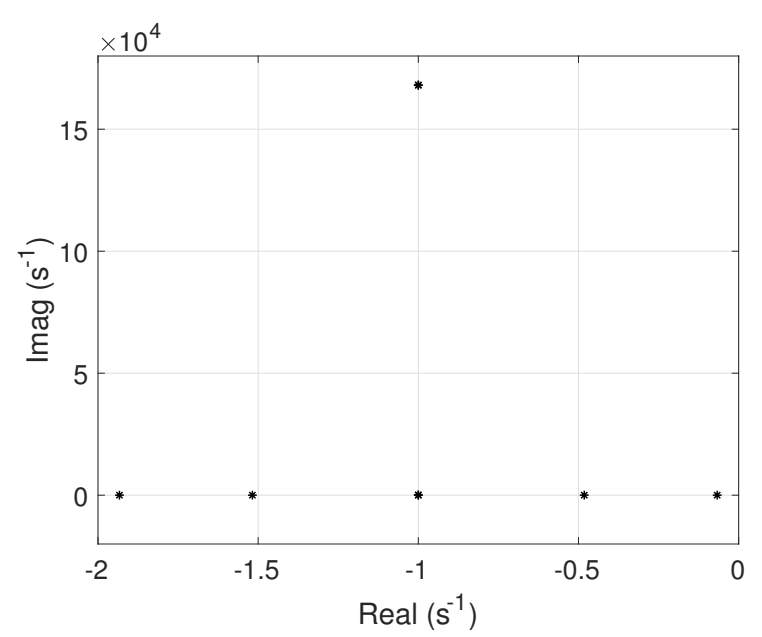

Fig. 1 Eigenvalues of the propagator matrix $\mathbf{M}$ at $22 \mathrm{~Hz}$.

\section{Numerical scheme}

In absence of a forcing function, the poroelastic system of equations (25) can be expressed as

$\mathbf{q}_{\mathbf{t}}=\mathbf{M q}$

where $\mathbf{M}$ is the propagator matrix, containing the material properties and spatial derivative operators. The eigenvalues of $\mathbf{M}$ come in conjugate pairs. For an inviscid pore fluid $(\eta=0)$, the eigenvalues of $\mathbf{M}$ lie along the imaginary axis, which implies the absence of dissipation from the system. On the other hand, for the viscous pore fluid $(\eta \neq 0)$, the eigenvalues of $\mathbf{M}$ also contain a negative real part. Furthermore, a substantial difference between the magnitude of the real part in eigenvalues of different wave modes in the system causes it to be stiff. Figure 1 shows the representative eigenvalues of (46) computed for an isotropic sandstone with material properties given in Table 1. Stiffness in the system causes instabilities in explicit time integration schemes unless a very small time step is used. Alternatively, an implicit time integration scheme can be an unconditionally stable, but not efficient for a linear hyperbolic system. Carcione and co-workers have used an explicit approach in time operator splitting, also known as Godunov splitting or fractional step [8, $9,11]$, which separates the dissipative term from the conservation term at each time step. In our work we solve the stiff (dissipative) part of the system analytically and the non-stiff (conservation) part by using a nodal DG method, paired with a $4^{\text {th }}$-order low-storage explicit Runge-Kutta scheme (LSERK) [20]. Equation (25) is split into stiff and non-stiff part as follows: Stiff part:

$\partial_{t} \mathbf{q}=\mathbf{D q}$

Non-stiff part:

$\partial_{t} \mathbf{q}+\nabla \cdot\left(\mathcal{A}_{n} \mathbf{q}\right)=\mathbf{f}(\mathbf{t})$.

At each time step, equation (47), which is a simple ordinary differential equation, is solved analytically [9] and these intermediate solutions are plugged into (48) as an initial solution. The analytical solution of equation (47) is given in Appendix A. The numerical solution of (48) is computed using the nodal DG finite-element method, discussed next.

4.1 Nodal discontinuous Galerkin scheme for the poroelastic system

We consider that the domain $\Omega$ is Lipschitz and triangulated by $D^{k}$ elements, where each element $D^{k}$ is the image of a reference element $\hat{D}$ under the local mapping

$\mathbf{x}^{\mathbf{k}}=\Phi \hat{\mathbf{x}}$

where $\mathbf{x}^{\mathbf{k}}$ and $\hat{\mathbf{x}}$ denote the physical and reference coordinates on $D^{k}$ and $\hat{D}$, respectively. The approximate local solution over each element is expressed as

$V_{h}\left(D^{k}\right)=\Phi^{k} \circ V_{h}(\hat{D})$,

where $V_{h}\left(D^{k}\right)$ and $V_{h}(\hat{D})$ represent the approximation spaces for the physical and the reference element, respectively.

The global solution space $V_{h}\left(\Omega_{h}\right)$ is defined as the direct sum of the local approximation spaces

$V_{h}\left(\Omega_{h}\right)=\bigoplus_{D^{k}} V_{h}\left(D^{k}\right)$

In this work, we take $V_{h}(\hat{D})=P^{N}(\hat{D})$, where $P^{N}(\hat{D})$ is the polynomial space of total degree $N$ on the reference element. 
Table 2 Material properties for several poroelastic media used in the examples $[9,13]$

\begin{tabular}{ccccc}
\hline Properties & $\begin{array}{c}\text { Sandstone } \\
\text { (Orthotropic) }\end{array}$ & $\begin{array}{c}\text { Epoxy-glass } \\
\text { (Orthotropic) }\end{array}$ & $\begin{array}{c}\text { Sandstone } \\
\text { (Isotropic) }\end{array}$ & $\begin{array}{c}\text { Shale } \\
\text { (Isotropic) }\end{array}$ \\
\hline$K_{s}(\mathrm{GPa})$ & 80 & 40 & 40 & 7.6 \\
$\rho_{s}\left(\mathrm{~kg} / \mathrm{m}^{3}\right)$ & 2500 & 1815 & 2500 & 2210 \\
$c_{11}(\mathrm{GPa})$ & 71.8 & 39.4 & 36 & 11.9 \\
$c_{12}(\mathrm{GPa})$ & 3.2 & 1.2 & 12 & 3.96 \\
$c_{13}(\mathrm{GPa})$ & 1.2 & 1.2 & 12 & 3.96 \\
$c_{33}(\mathrm{GPa})$ & 53.4 & 13.1 & 36 & 11.9 \\
$c_{55}(\mathrm{GPa})$ & 26.1 & 3 & 12 & 3.96 \\
$\phi$ & 0.2 & 0.2 & 0.2 & 0.16 \\
$\kappa_{1}\left(10^{-15} \mathrm{~m}^{2}\right)$ & 600 & 600 & 600 & 100 \\
$\kappa_{3}\left(10^{-15} \mathrm{~m}^{2}\right)$ & 100 & 100 & 600 & 100 \\
$T_{1}$ & 2 & 2 & 2 & 2 \\
$T_{3}$ & 3.6 & 3.6 & 2 & 2 \\
$K_{f}(\mathrm{GPa})$ & 2.5 & 2.5 & 2.5 & 2.5 \\
$\rho_{f}\left(\mathrm{Kg} / \mathrm{m}^{3}\right)$ & 1040 & 1040 & 1040 & 1040 \\
$\eta\left(10^{-3} \mathrm{Kg} / \mathrm{m} . \mathrm{s}\right)$ & 1 & 1 & 1 & 1 \\
$\lambda^{*}(\mathrm{~m} / \mathrm{s})$ & 6000 & 5240 & 4250 & 2480 \\
\hline
\end{tabular}

${ }^{*}$ computed in this study.

Let $f$ be the face of element $D^{k}$ with neighboring element $D^{k,+}$ and unit outward normal vector $\mathbf{n}$. Let $u$ be a function which is discontinuous across the element interface. The interior value $u^{-}$and exterior value $u^{+}$ on a face $f$ of $D^{k}$ are defined as

$u^{-}=\left.u\right|_{f \bigcap \partial D^{k}}, \quad u^{+}=\left.u\right|_{f \bigcap \partial D^{k,+}}$.

Jump and average of a scalar function $u$ over $f$ is defined as

$$
[[u]]=u^{+}-u^{-}, \quad\{\{u\}\}=\frac{u^{+}+u^{-}}{2} .
$$

The jump and average of a vector valued functions are computed component wise.

The nodal DG scheme for (48) can be constructed by multiplying (48) with a basis function $\mathbf{p} \in P^{N}(\hat{D})$ and integrating by parts twice

$$
\begin{aligned}
\int_{D^{k}}\left(\partial_{t} \mathbf{q}_{\mathbf{h}} \mathbf{p}\right. & \left.+\nabla \cdot\left(\mathcal{A}_{n} \mathbf{q}_{\mathbf{h}}\right) \mathbf{p}\right) d \mathbf{x} \\
& +\int_{\partial D^{k}}\left(\left(\mathcal{A}_{n} \mathbf{q}_{\mathbf{h}}\right)^{*}-\left(\mathcal{A}_{n} \mathbf{q}_{\mathbf{h}}\right)^{-}\right) \mathbf{p} d \mathbf{x} \\
& =\int_{D^{k}} \mathbf{f} \mathbf{p} d \mathbf{x},
\end{aligned}
$$

where $\mathbf{q}_{\mathbf{h}}$ is the discretized solution and $\left(\mathcal{A}_{n} \mathbf{q}_{\mathbf{h}}\right)^{*}$ represents the numerical flux.

Equation (52) represents the numerical scheme in strong form and thus does not assure any smoothness on the basis function $\mathbf{p}$. To compute the basis function $\mathbf{p}$, we have utilized the nodal basis function approach, discussed in the following section.

\subsection{Nodal basis function}

The discretized solution $\mathbf{q}_{\mathbf{h}}$ in (48) follows a componentwise expansion into $N_{p}=N_{d o f}^{D^{k}}=N_{d o f}^{D^{k}}(N)$ nodal trial basis function of order $N$ [20],

$\mathbf{q}_{\mathbf{h}}(\mathbf{x}, t)=\bigoplus_{D^{k}} \sum_{n=1}^{N_{p}} \mathbf{q}_{\mathbf{h}, \mathbf{n}}^{\mathbf{D}^{\mathbf{k}}}(t) p_{n}(\mathbf{x})$.

Here $\mathbf{q}_{\mathbf{h}, \mathbf{n}}^{\mathbf{D}^{\mathbf{k}}}$ indicates the local expansion of $\mathbf{q}_{\mathbf{h}}$ within element $D^{k}, p_{n}(\mathbf{x})$ is a set of 2-D Lagrange polynomials associated with the nodal points, $\left\{x_{n}\right\}_{n=1}^{N_{p}}$. The explicit expression for computing the Lagrange polynomials in $2 \mathrm{D}$ space is not known, but can be constructed by expressing the approximated solution in modal and nodal form, simultaneously. Expressing the $\mathbf{q}_{\mathbf{h}, \mathbf{n}}^{\mathbf{D}^{\mathbf{k}}}$ in modal and nodal form simultaneously, yields

$\mathcal{V}^{T} p_{n}(\mathbf{x})=\mathbf{P}_{\mathbf{n}}(\mathbf{x})$,

where $\mathcal{V}$ is the Vandermonde matrix of basis functions, used for approximating the modal form $\mathbf{q}_{\mathbf{h}, \mathbf{n}}^{\mathbf{D}^{\mathbf{k}}}$ and $\mathbf{P}_{\mathbf{n}}(\mathbf{x})$ is a $2 \mathrm{D}$ orthonormalized basis function, constructed from Jacobi polynomials.

We have used the warp and blend method [20] to determine the coordinates of the nodal points in a triangle; for order $N$ interpolation, there are $N_{p}=\frac{(N+1)(N+2)}{2}$ such nodes.

\subsection{Numerical flux}

The numerical flux $\left(\mathcal{A}_{n} q_{h}\right)^{*}$ in (52) determines the unique solution at the shared edges of two elements. In this 


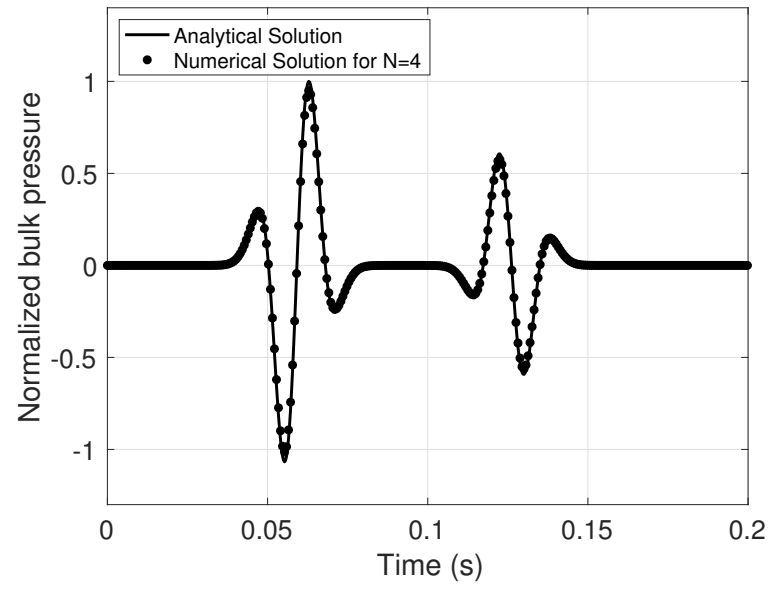

(a) Bulk pressure at $\eta=0, f_{c}=22 \mathrm{~Hz}$

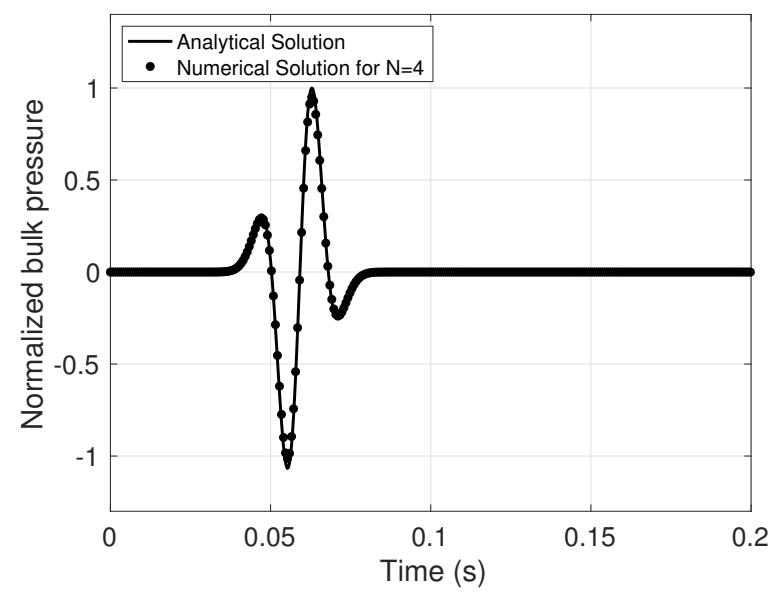

(c) Bulk pressure at $\eta \neq 0, f_{c}=22 \mathrm{~Hz}$

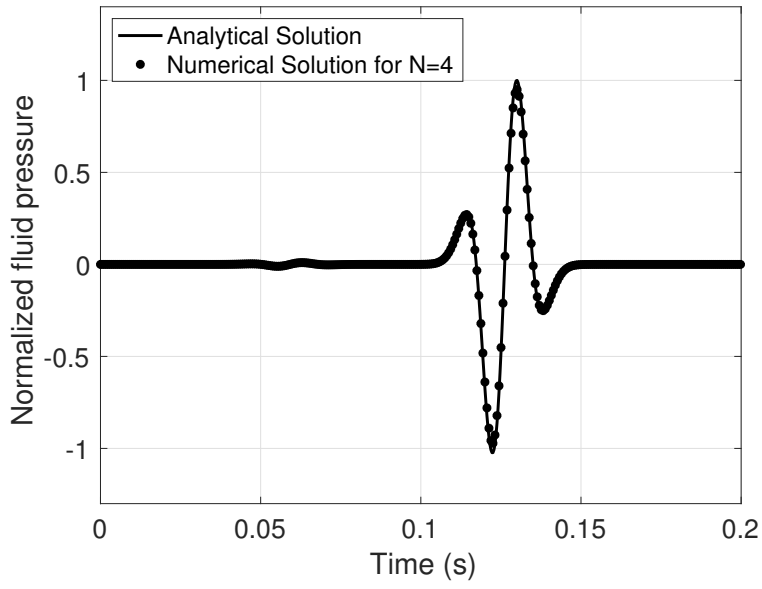

(b) Fluid pressure at $\eta=0, f_{c}=22 \mathrm{~Hz}$

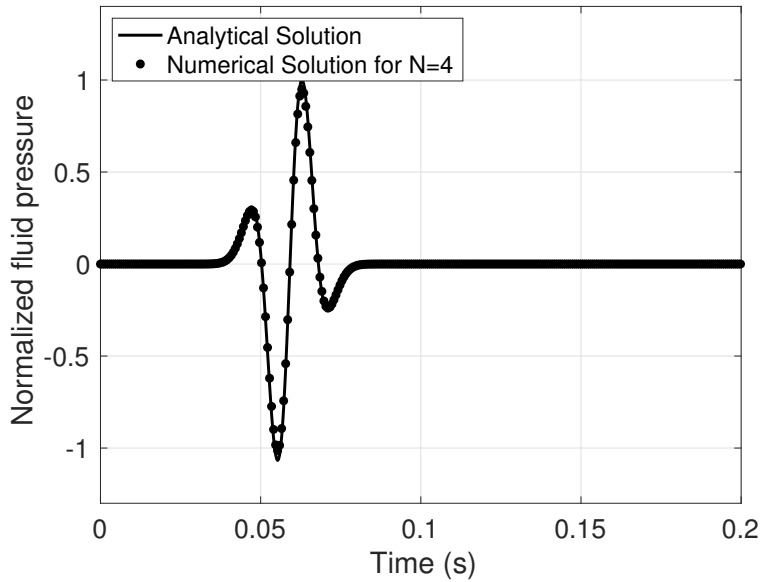

(d) Fluid pressure at $\eta \neq 0, f_{c}=22 \mathrm{~Hz}$

Fig. 2 A comparison between the analytical and the numerical solutions, computed in a poroacoustic media, at source-receiver offset of $250 \mathrm{~m}$, and $f_{c}=22 \mathrm{~Hz}$, where (a) normalized bulk pressure $(\eta=0)$, (b) normalized fluid pressure, computed with $(\eta=0)$, (c) normalized bulk pressure $(\eta \neq 0)$, and (d) normalized fluid pressure, computed with $(\eta \neq 0)$.

paper, we use the Lax-Friedrich flux [26] to compute $\left(\mathcal{A}_{n} q_{h}\right)^{*}$. The Lax-Friedrich flux is expressed as

$$
\left(\mathcal{A}_{n} q_{h}\right)^{*}=\left\{\left\{\mathcal{A}_{n} q_{h}\right\}\right\}+\frac{\lambda}{2}\left[\left[q_{h}\right]\right],
$$

where $\lambda$ is the maximum speed of the waves in the system. Substituting (55) into (52), we recover the final numerical scheme in strong form as

$$
\begin{aligned}
\int_{D^{k}}\left(\partial_{t} \mathbf{q}_{\mathbf{h}} \mathbf{p}\right. & \left.+\nabla \cdot\left(\mathcal{A}_{n} \mathbf{q}_{\mathbf{h}}\right) \mathbf{p}\right) d \mathbf{x} \\
& +\int_{\partial D^{k}}\left(\left[\left[\mathcal{A}_{n} \mathbf{q}_{\mathbf{h}}\right]\right]+\frac{\lambda}{2}\left[\left[q_{h}\right]\right]\right) \mathbf{p} d \mathbf{x} \\
& =\int_{D^{k}} \mathbf{f} \mathbf{p} d \mathbf{x} .
\end{aligned}
$$

In order to compute $\lambda$, the maximum speed of the wave in the system, we have used a plane-wave approach [9]. A detailed formulation for computing $\lambda$ is given in Appendix B.

\subsection{Time discretization}

In the present study, we have employed the five-steps low-storage explicit Runge-Kutta (LSERK) method [6]. LSERK is preferred over other methods as it saves memory at the cost of computation time. A stable CFL condition depending on the polynomial degree $N$ is derived by Cockburn and Shu [6] and employed here. 


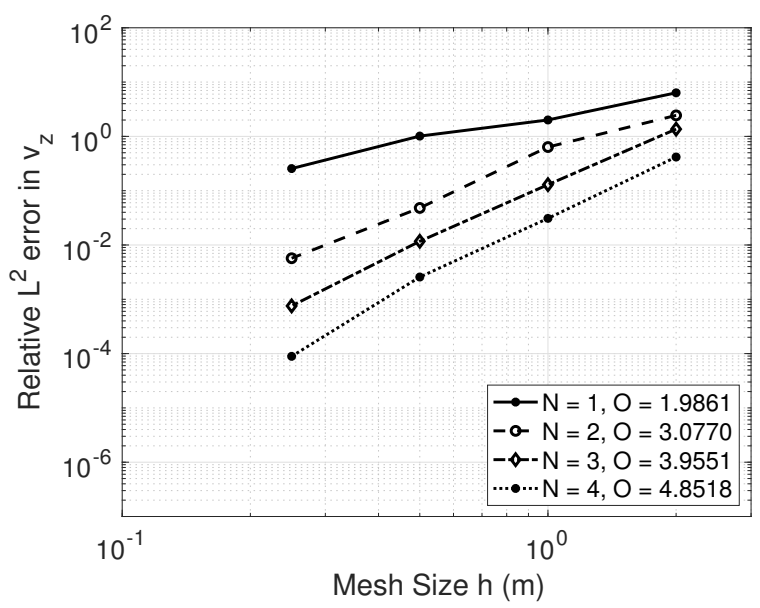

(a) Inviscid $\operatorname{case}(\eta=0)$

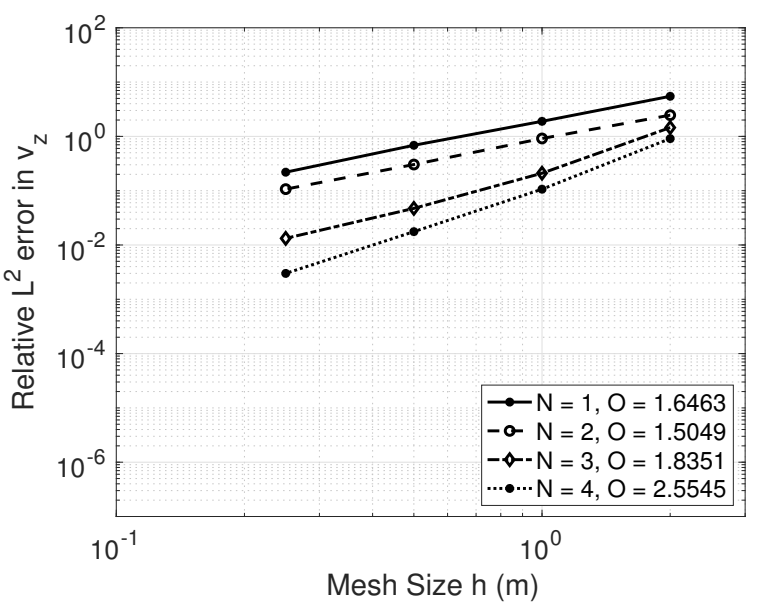

(b) Viscid $\operatorname{case}(\eta \neq 0)$

Fig. $3 L^{2}$ error of the solid particle velocity $\left(v_{z}\right)$ (in a plane wave) as a function of the mesh size $h$ computed at $N=$ $1,2,3$ and 4 for (a) inviscid case $\eta=0$ and (b) viscid case $\eta \neq 0$.

\subsection{Variational crime}

In many applications, the external forcing function $\mathbf{f}$ is considered as a point source or Dirac function. A Dirac delta function is not $L^{2}$ integrable and the term $\int_{D^{k}} \mathbf{f} \mathbf{p} d \mathbf{x}$ in (56) is not well defined. Thus we commit a variational crime while evaluating the $\mathbf{f}(\mathbf{x})=$ $\delta\left(\mathbf{x}-\mathbf{x}_{\mathbf{0}}\right)$. A point source approximation is numerically implemented as

$\sum_{D^{k}} \int_{D^{k}} \delta\left(\mathbf{x}-\mathbf{x}_{\mathbf{0}}\right) \cdot \mathbf{p}=\int_{\Omega} \mathbf{p} \cdot \delta\left(\mathbf{x}-\mathbf{x}_{\mathbf{0}}\right)=\mathbf{p}\left(\mathbf{x}_{\mathbf{0}}\right)$.

\section{Computational experiments}

In this section, we illustrate the accuracy of our numerical scheme by comparing the analytical solution with the numerical solution and investigate the convergence. To check the accuracy between the numerical and the analytical solutions, we started our computational experiments with a poroacoustic system [8], which is a simplified poroelastic system, obtained by setting the solid rigidity to zero. Thus poroacoustic simulation only models the dilatational deformation. The properties of a poroacoustic media have been used to to describe the kinematics of emulsions and gels [21]. A system of equations describing the poroacoustic wave equation is given in Appendix C.
5.1 Poroacoustic medium: Comparison of analytical and numerical solutions

The analytical solution of a point source in a $2 \mathrm{D}$ homogeneous poroacoustic medium is given by Carcione and Quiroga-Goode [8] and implemented here to evaluate the quality of the solution obtained from our nodal DG scheme. The forcing function $\mathbf{f}$ is the product of Dirac's delta in space and Ricker's wavelet in time, which is expressed as

$f(t)=\exp \left[-\frac{1}{2} f_{c}^{2}\left(t-t_{0}\right)^{2}\right] \cos \left[\pi f_{c}\left(t-t_{0}\right)\right]$,

where $f_{c}$ is the source central frequency of the source and $t_{0}=3 / f_{c}$ is the wavelet delay.

Figure 2(a) and 2(b) present a comparison between the analytical and the numerical solutions of the bulk and the fluid pressure, computed at $22 \mathrm{~Hz}$ for an inviscid case $(\eta=0)$. We have used a polynomial degree $N=4$. Table 1 shows the material properties of the poroacoustic medium used, an acoustic version of a brine saturated sandstone. Figure 2(a) and 2(b) show a good agreement between solutions with an $L^{2}$ error of $0.04 \%$. Figure 2(c) and $2(\mathrm{~d})$ represent a comparison between the solutions of the bulk and the fluid pressure, computed at $22 \mathrm{~Hz}$ for a viscid case $(\eta \neq 0)$. Figure $2(\mathrm{c})$ and $2(\mathrm{~d})$ show a good agreement with an $L^{2}$ error of $0.05 \%$.

\subsection{Poroelastic medium: Convergence test}

An analytical solution for plane waves of the poroelastic system (25) is given by [11] and [13]. We implemented 


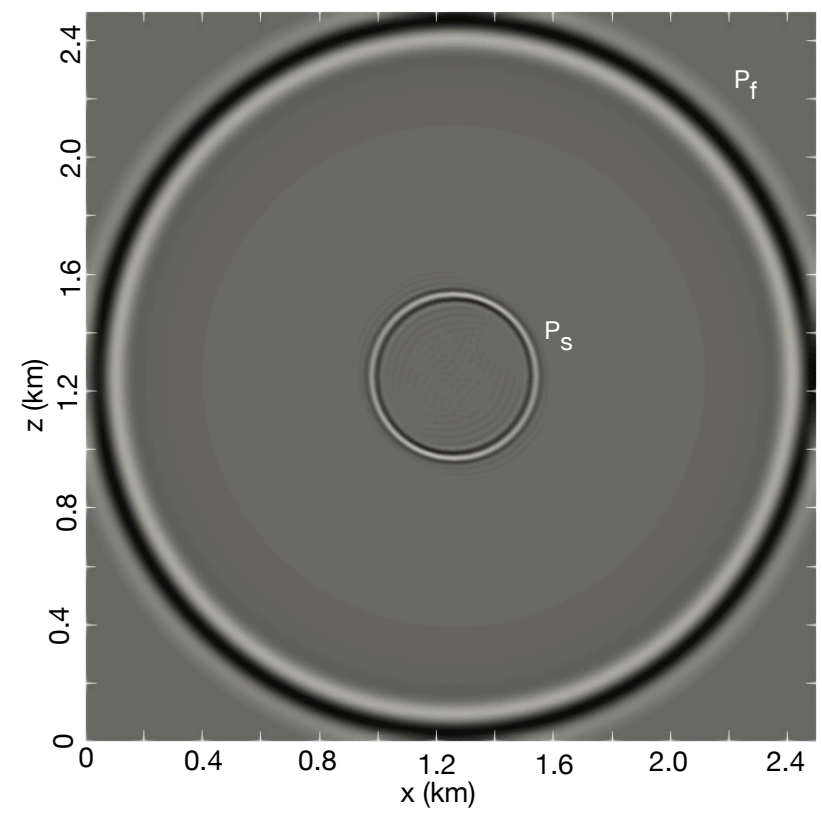

(a) Bulk pressure at $\eta=0, f_{c}=22 \mathrm{~Hz}$

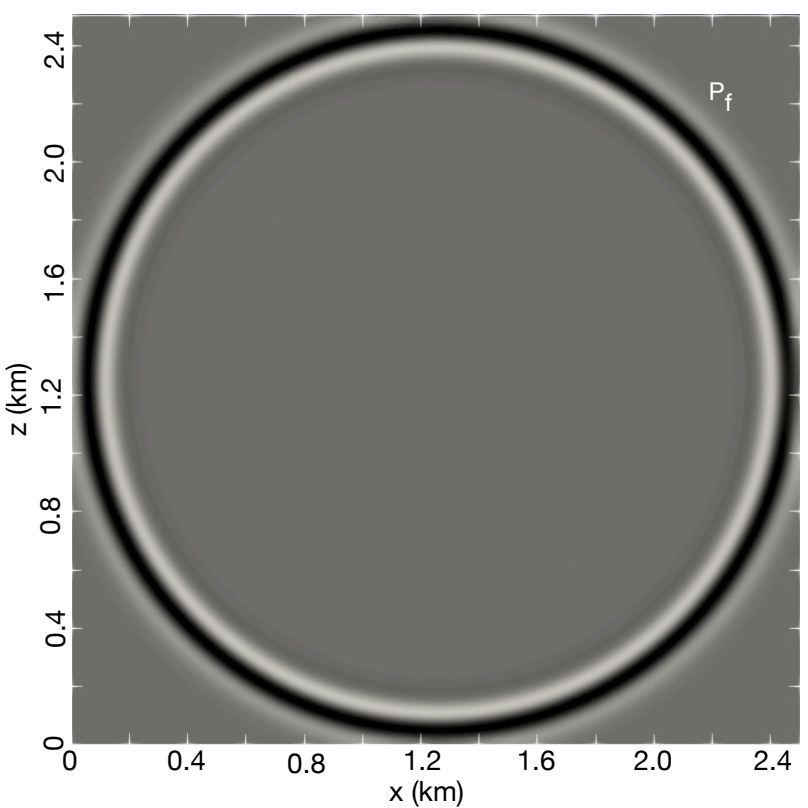

(c) Bulk pressure at $\eta \neq 0, f_{c}=22 \mathrm{~Hz}$

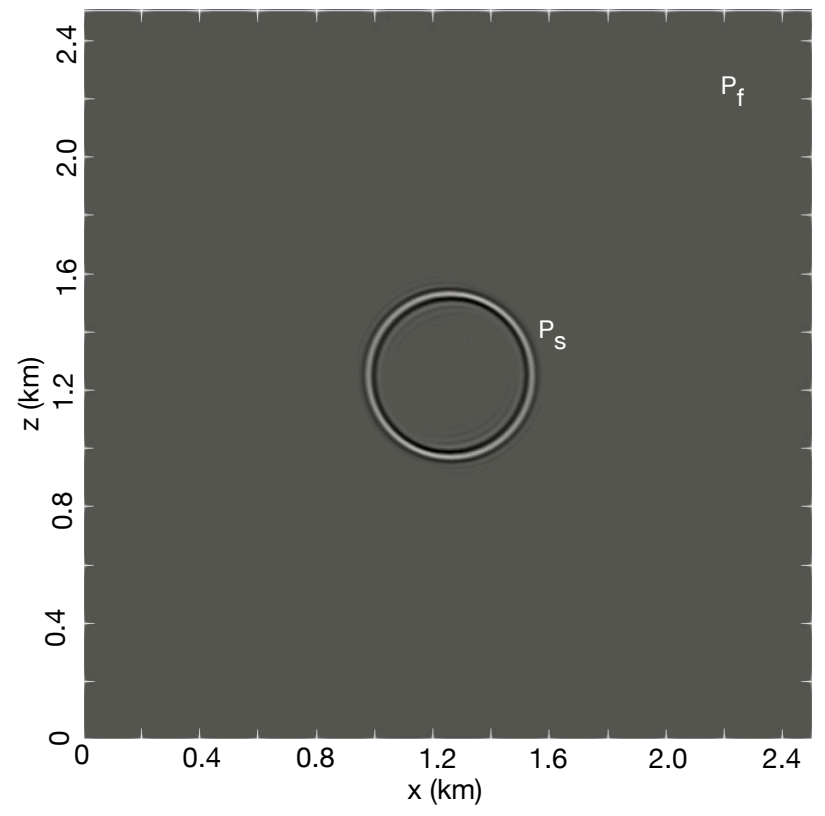

(b) Fluid pressure at $\eta=0, f_{c}=22 \mathrm{~Hz}$

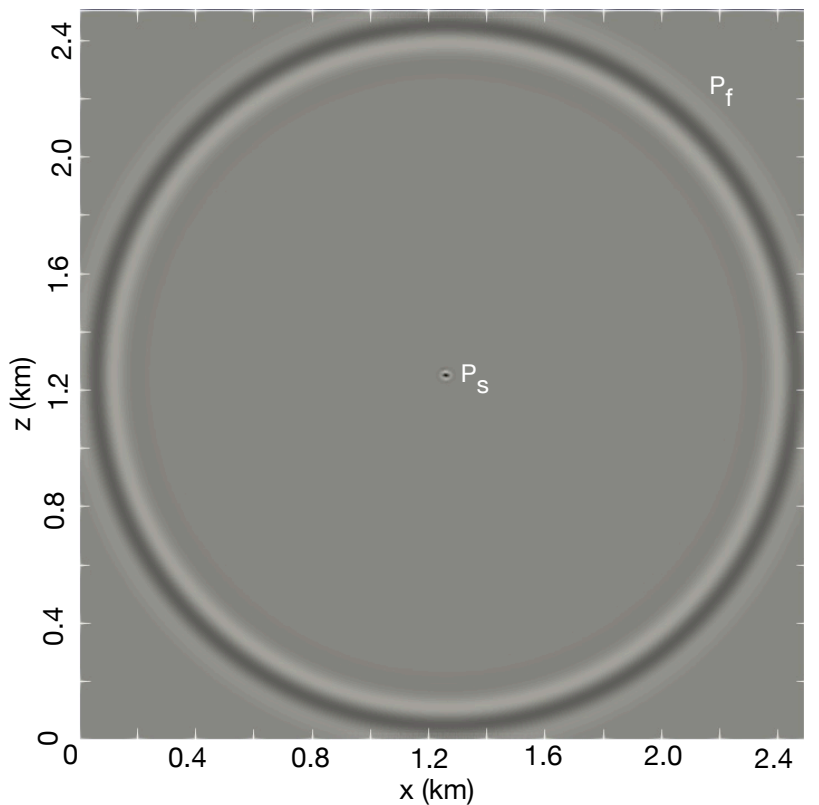

(d) Fluid pressure at $\eta \neq 0, f_{c}=22 \mathrm{~Hz}$

Fig. 4 Snapshots of (a) bulk pressure $p$ (b) fluid pressure $p_{f}$ for the inviscid case $(\eta=0)$, computed at $t=0.36 \mathrm{~s}$. Snapshots of (c) bulk pressure $p(\mathrm{~d})$ fluid pressure for viscid case $(\eta \neq 0)$, computed at $t=0.36 \mathrm{~s}$. The forcing function is a bulk source (energy is partitioned between solid and fluid) with a central frequency of $22 \mathrm{~Hz}$. Numerical solution is computed for a polynomial of order $4 . \mathrm{P}_{\mathrm{f}}$ : Fast compressional wave, $\mathrm{P}_{\mathrm{s}}$ : slow wave (Biot mode). 


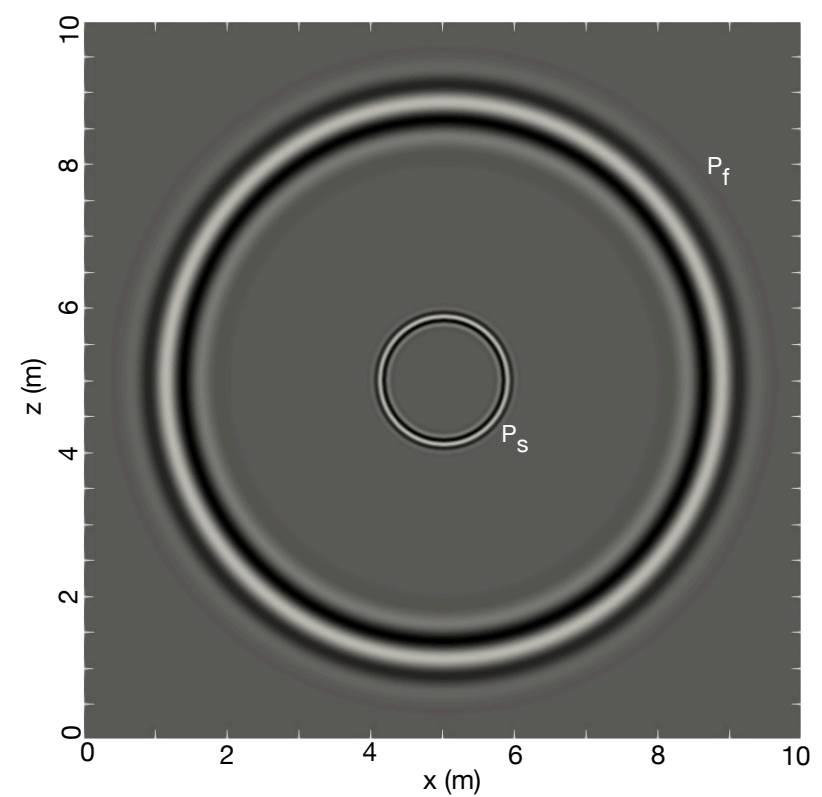

(a) Bulk pressure at $\eta=0, f_{c}=4.5 \mathrm{kHz}$

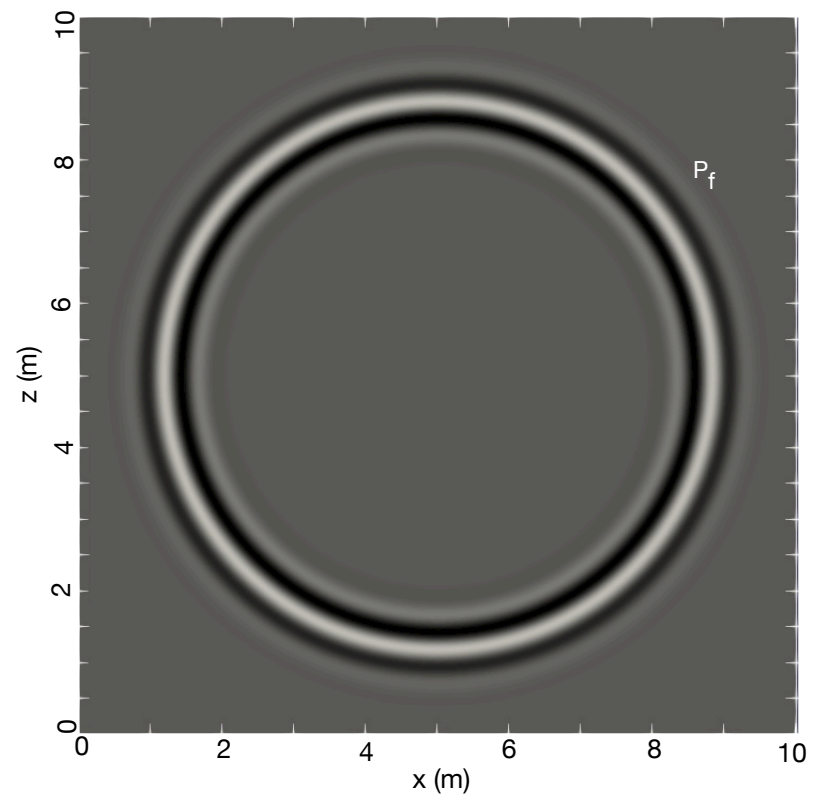

(c) Bulk pressure at $\eta \neq 0, f_{c}=4.5 \mathrm{kHz}$

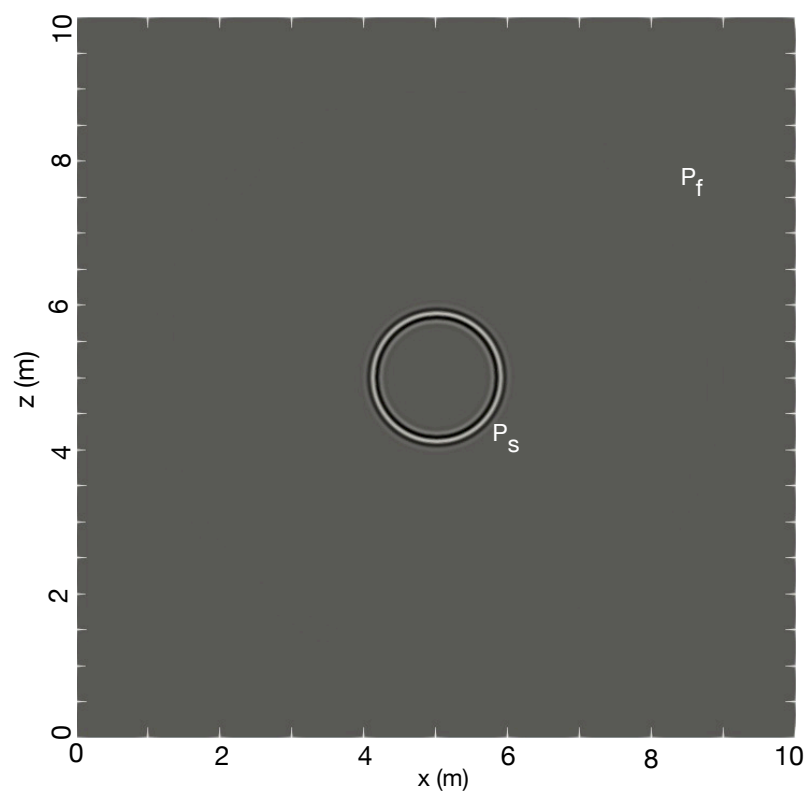

(b) Fluid pressure at $\eta=0, f_{c}=4.5 \mathrm{kHz}$

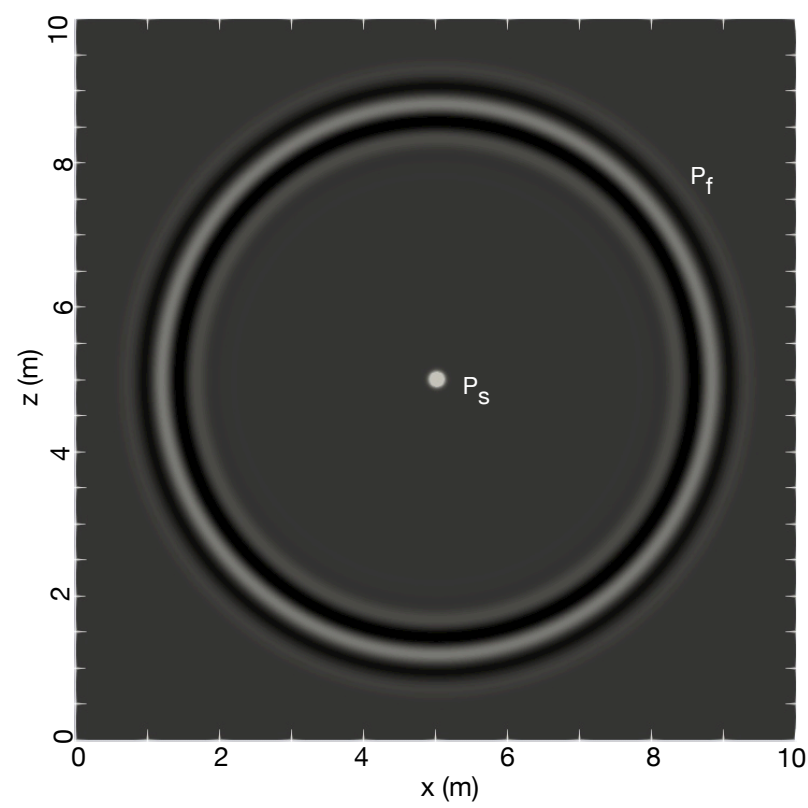

(d) Fluid pressure at $\eta \neq 0, f_{c}=4.5 \mathrm{kHz}$

Fig. 5 Snapshots of (a) bulk pressure $p$ (b) fluid pressure $p_{f}$ for the inviscid case $(\eta=0)$, computed at $t=1.2$ ms. Snapshots of (c) bulk pressure $p$ (d) fluid pressure for viscid case $(\eta \neq 0)$, computed at $t=1.2 \mathrm{~ms}$. The forcing function is bulk source (energy is partitioned between solid and fluid) with a central frequency of $22 \mathrm{~Hz}$. Numerical solution is computed for a polynomial of order $4 . \mathrm{P}_{\mathrm{f}}$ : Fast compressional wave, $\mathrm{P}_{\mathrm{s}}$ : slow wave (Biot mode).

the solution computed by de la Puente et al. [13] to test the convergence of the numerical scheme in (56). The convergence analysis is performed in both regimes, non-stiff and stiff, with a periodic boundary condition. The convergence is computed for brine-filled (viscid and inviscid) isotropic sandstone. The properties are given in Table 2. The CFL value is 0.4 for the computation. Figure 3(a) shows a convergence plot of the $L^{2}$ error of $v_{z}$ in the non-stiff regime. The rate of convergence shows an order of $O\left(h^{N+1}\right)$. Figure 3(b) represents the convergence plot of the $L^{2}$ error of $v_{z}$ in the stiff regime. The convergence reported in Figure 3 are the minimum 


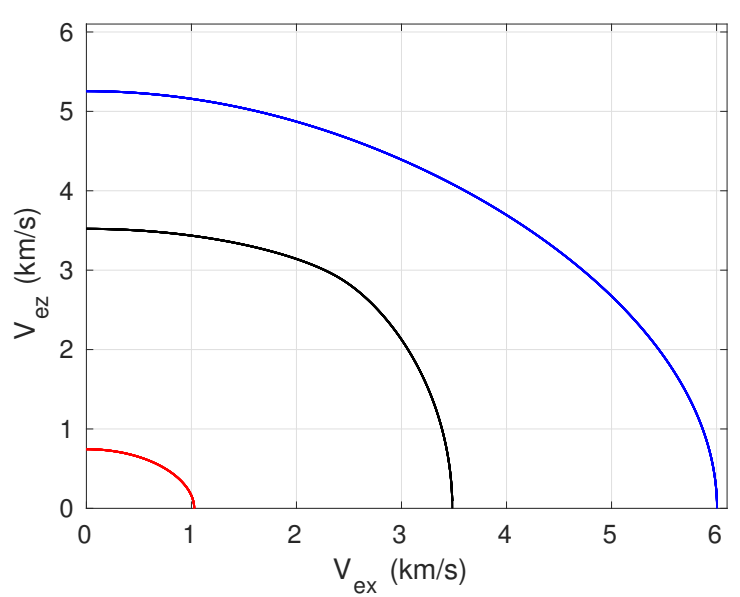

(a) Orthotropic sandstone

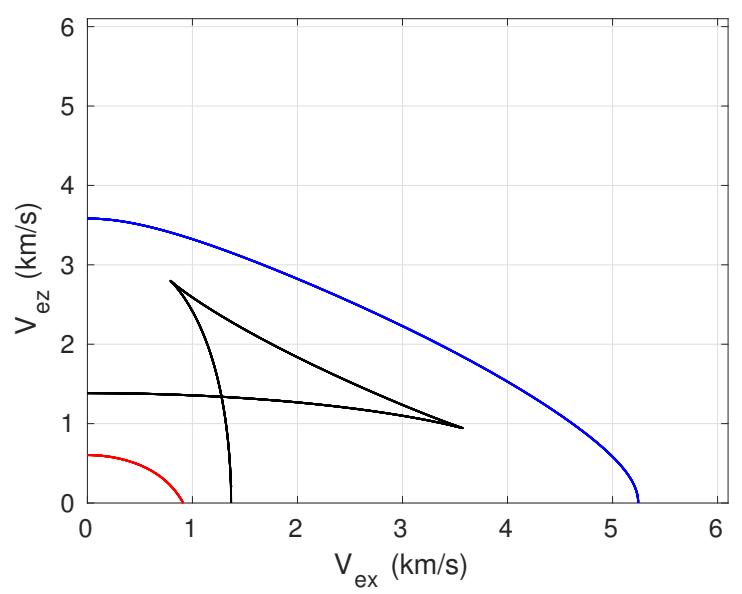

(c) Epoxy-glass

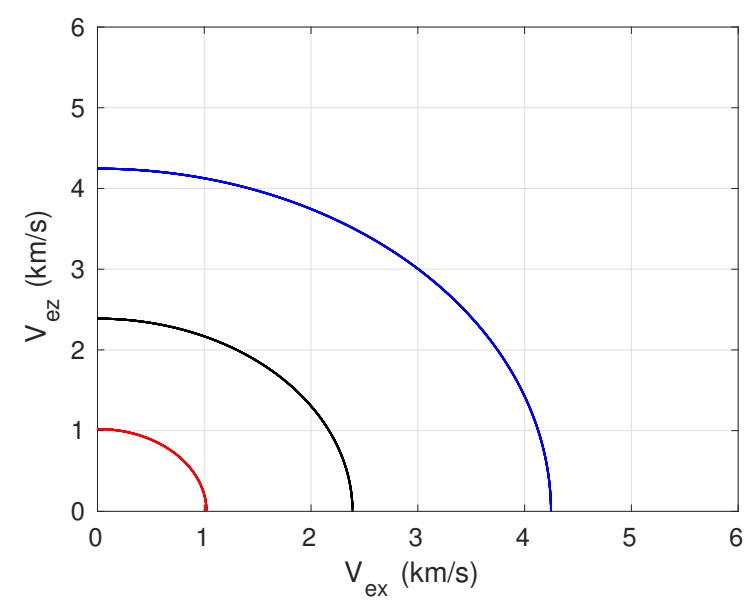

(b) Isotropic sandstone

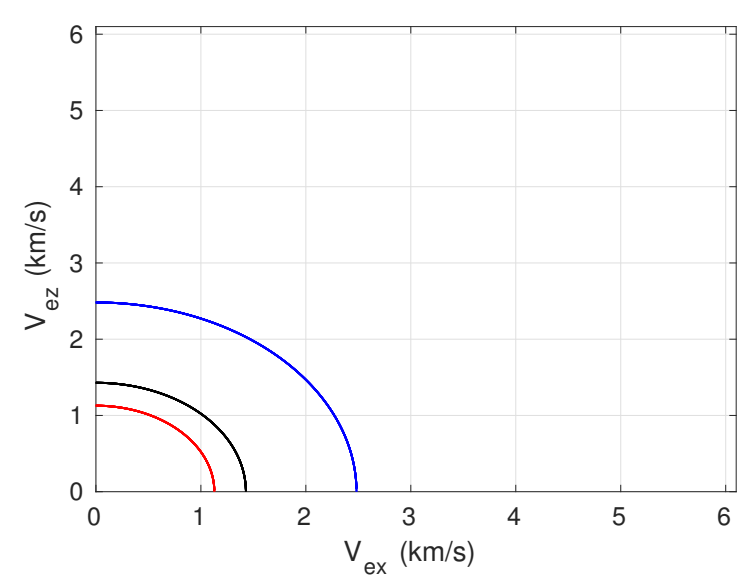

(d) Isotropic shale

Fig. 6 Energy velocity surface for $\eta=0$, computed in a $x-z$ plane for (a) orthotropic sandstone, (b) isotropic sandstone, (c) epoxy-glass and (d) isotropic shale. The material properties are given in Table 2. For homogenous media, the geometry of the energy velocity surfaces resembles the wavefronts of the compressional, shear, and slow $\mathrm{P}$ waves. $\mathrm{V}_{\text {ex }}$ and $\mathrm{V}_{\mathrm{ez}}$ are energy velocities in $x$ - and $z$ - directions, respectively.

rate of convergence. It is worth to note that the rate of convergence deteriorates due to the fact that operator splitting is a first-order accurate in time. For $N=1$, the rate of convergence is $\approx 2$, which is expected rate for a nodal DG scheme. For higher orders $(N=2,3,4)$ the convergence rate is dominated by the accuracy of the time integration scheme. In any case, the convergence rate is better than any low-order scheme.

\subsection{Homogeneous poroacoustic medium: Wave-field simulation}

We use the properties of the poroacoustic medium described in Table 1, which represents a brine-saturated sandstone. The Biot's characteristic frequency for this medium is $18 \mathrm{kHz}$. Thus, the system of equations (25) and the numerical scheme (56) used in this work is valid for a frequency of the forcing function (f) less than $18 \mathrm{kHz}$. We have performed the simulations for the in$\operatorname{viscid}(\eta=0)$ and viscid $(\eta \neq 0)$ cases at frequencies varying from the seismic to the sonic range. The forcing function considered here is given in (58) and located at the center of the computational domain.

Figure 4 shows the numerical results with a forcing function of central frequency $\left(f_{c}=22 \mathrm{~Hz}\right)$. The size of the computational domain is $2.5 \mathrm{~km} \times 2.5 \mathrm{~km}$. The minimum size of the edge of the equilateral triangles, used to mesh the domain, is $20 \mathrm{~m}$. Figure 5(a) and 5(b) represent the snapshots of bulk and fluid pressures, respectively, computed at $t=0.36 \mathrm{~s}$ and $\eta=0$ 


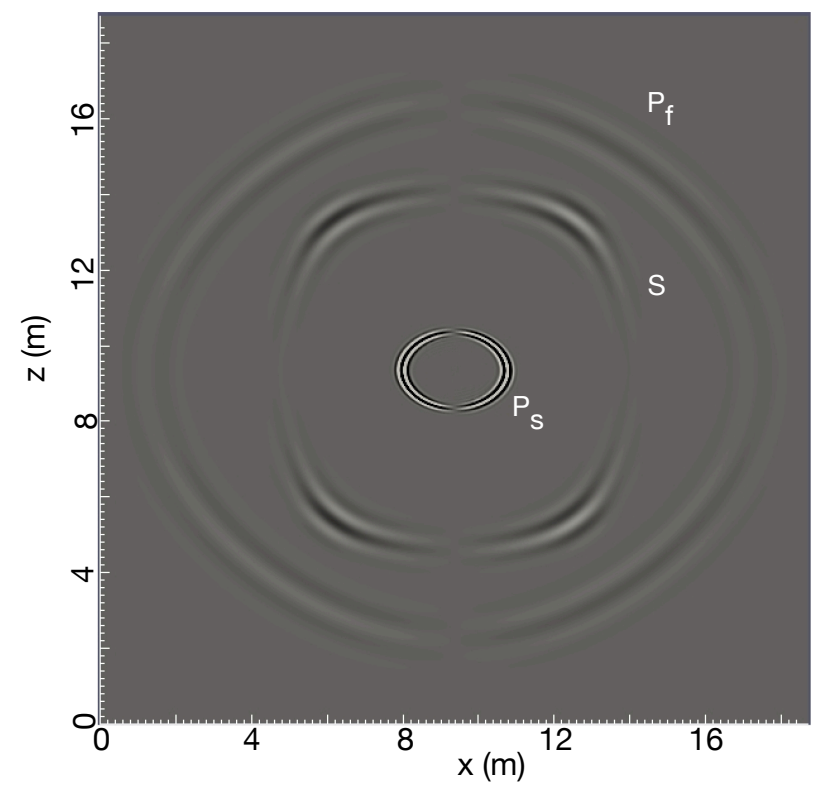

(a) $b_{x}$ at $\eta=0$

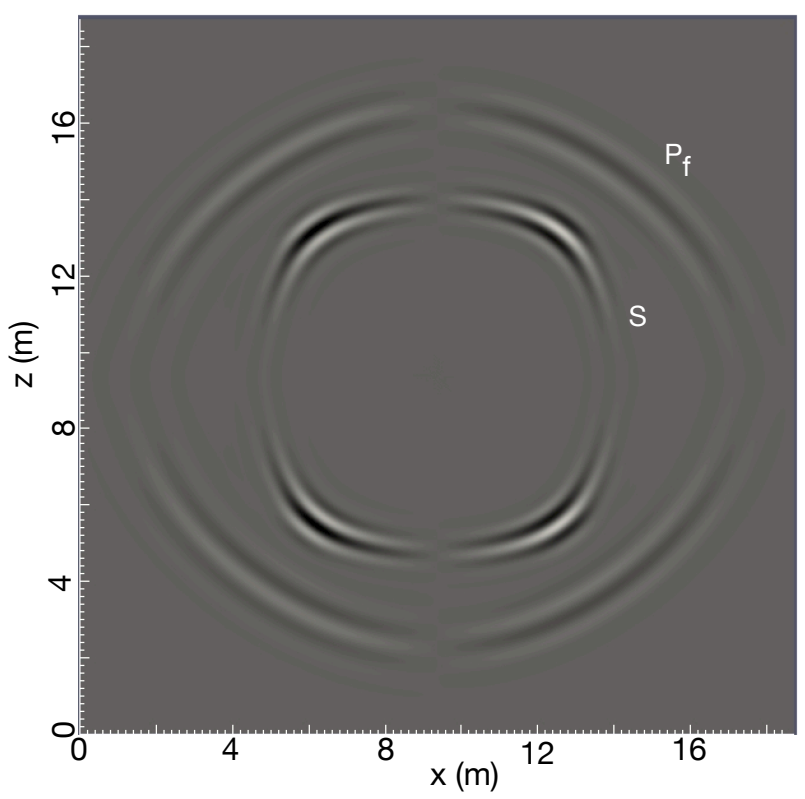

(c) $b_{x}$ at $\eta \neq 0$

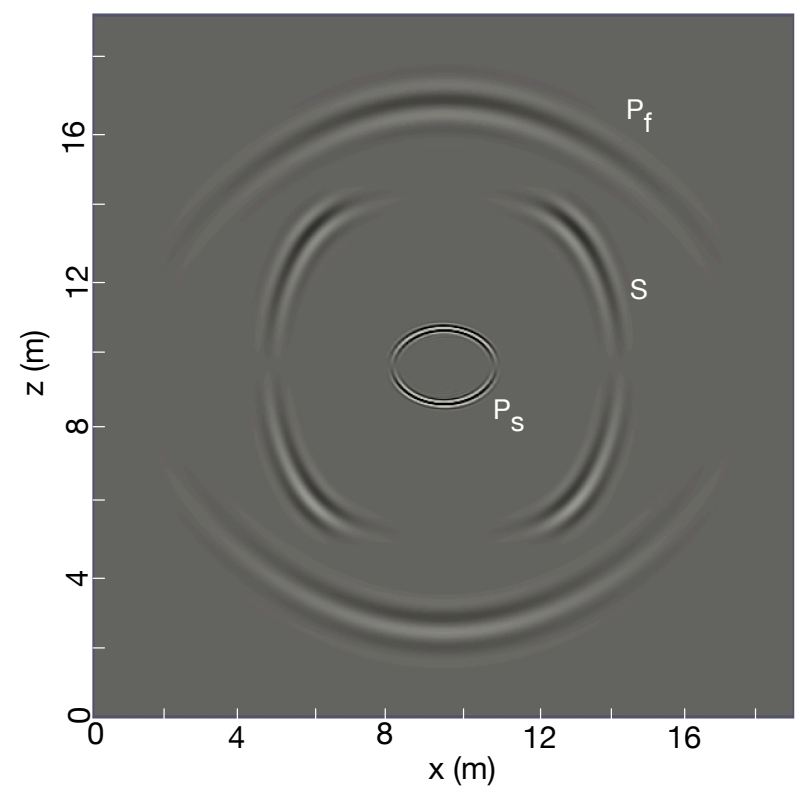

(b) $b_{z}$ at $\eta=0$

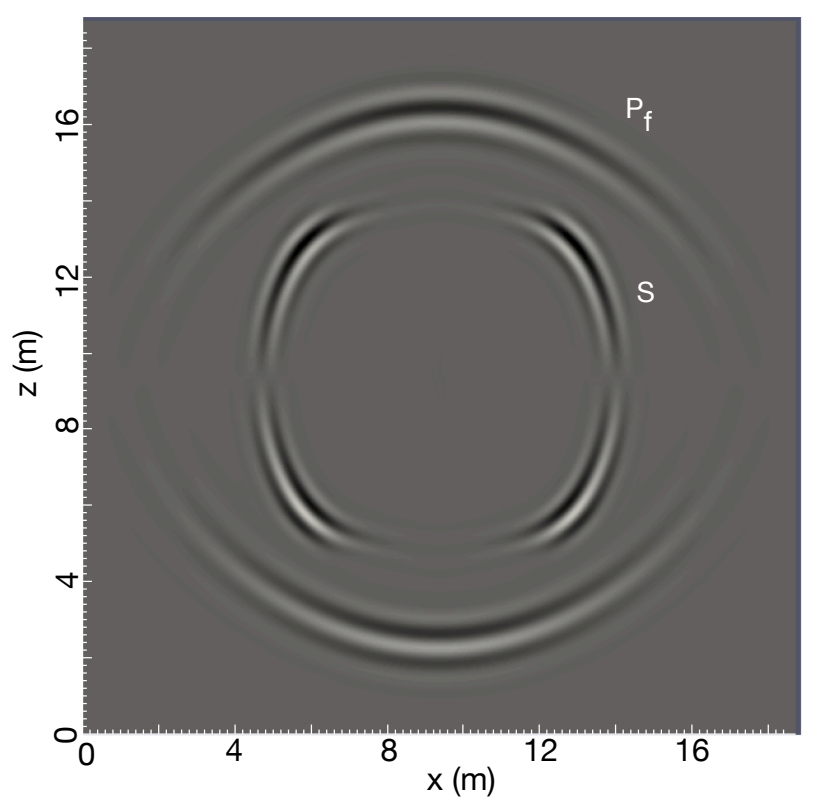

(d) $b_{z}$ at $\eta \neq 0$

Fig. 7 Snapshots of the centre of mass particle velocity in orthotropic sandstone, computed at $t=1.6 \mathrm{~ms}$, where (a) and (b) corresponds to $\eta=0$, and (c) and (d) corresponds to $\eta \neq 0$. The central frequency of the forcing function is $3730 \mathrm{~Hz}$. The solution is computed for a polynomial of order $4 . \mathrm{P}_{\mathrm{f}}$ : Fast compressional wave, $\mathrm{S}$ : Shear wave, $\mathrm{P}_{\mathrm{s}}$ : slow wave (Biot mode).

with a bulk forcing function. The bulk forcing function assumes that the energy is partitioned between the solid and fluid phases [8]. Figure 4(a) clearly shows both phases of $\mathrm{P}$ waves (fast and slow) whereas the fluid pressure in Figure 4(b) is dominated by a slow $\mathrm{P}$ wave, being the amplitude of the fast $\mathrm{P}$ wave very subtle. Since the results in Figure $4(\mathrm{a})$ and $4(\mathrm{~b})$ are simulated for the inviscid case, the slow $\mathrm{P}$ wave is not attenuated. Figure $4(\mathrm{c})$ and $4(\mathrm{~d})$ represents the bulk and fluid pressures at $t=0.36 \mathrm{~s}$ and $\eta \neq 0$. We remark that the slow $\mathrm{P}$ wave in Figure 4(c) and 4(d) attenuates faster than those in Figure 4(a) and 4(b). This is in agreement with the physics of Biot's theory, which states that for $\omega \leq \omega_{c}$, 


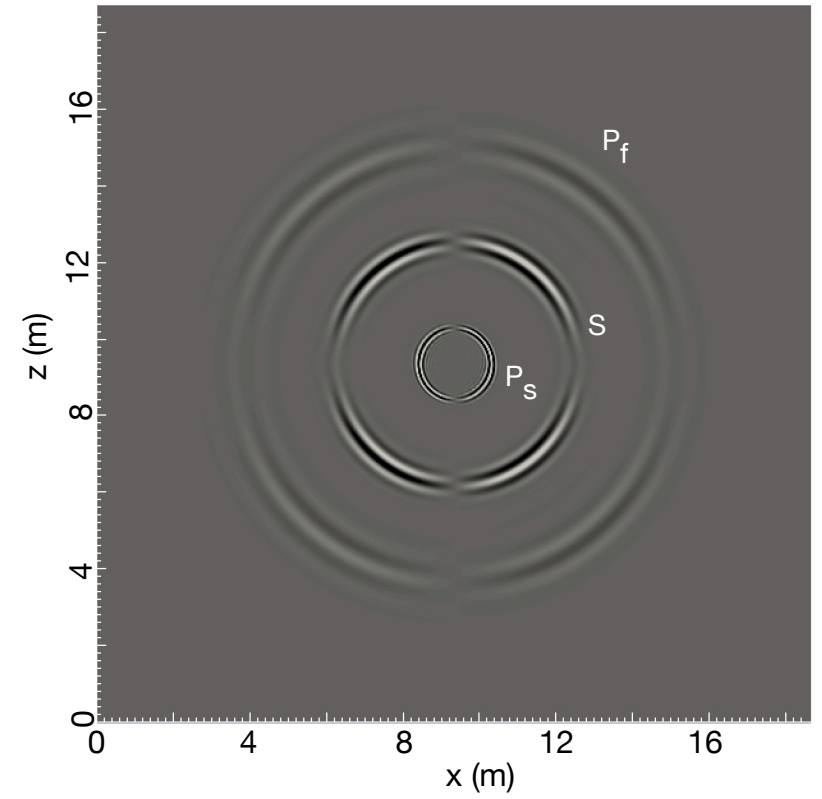

(a) $b_{x}$ at $\eta=0$

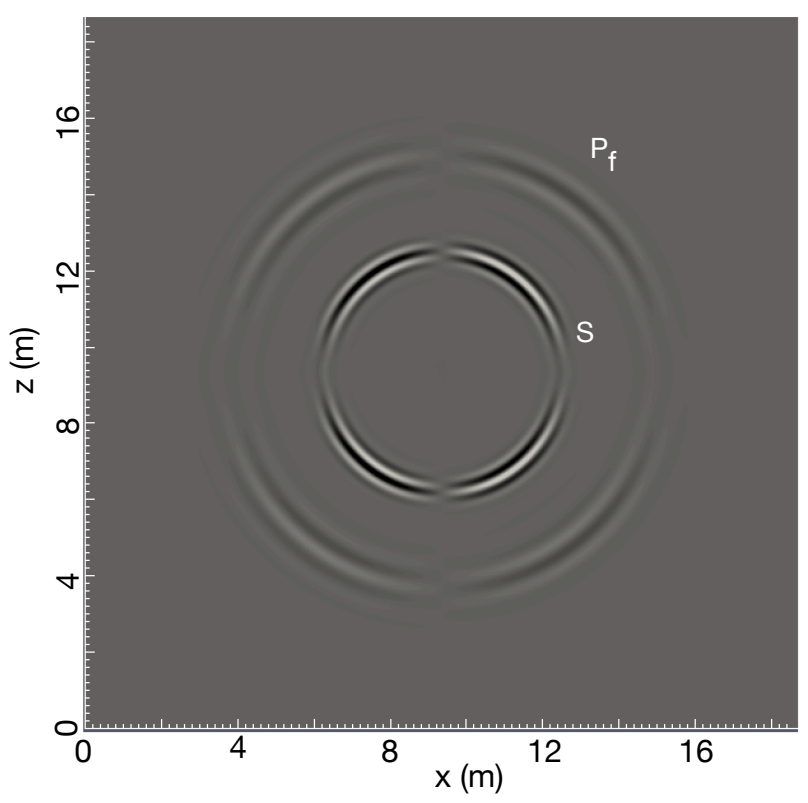

(c) $b_{x}$ at $\eta \neq 0$

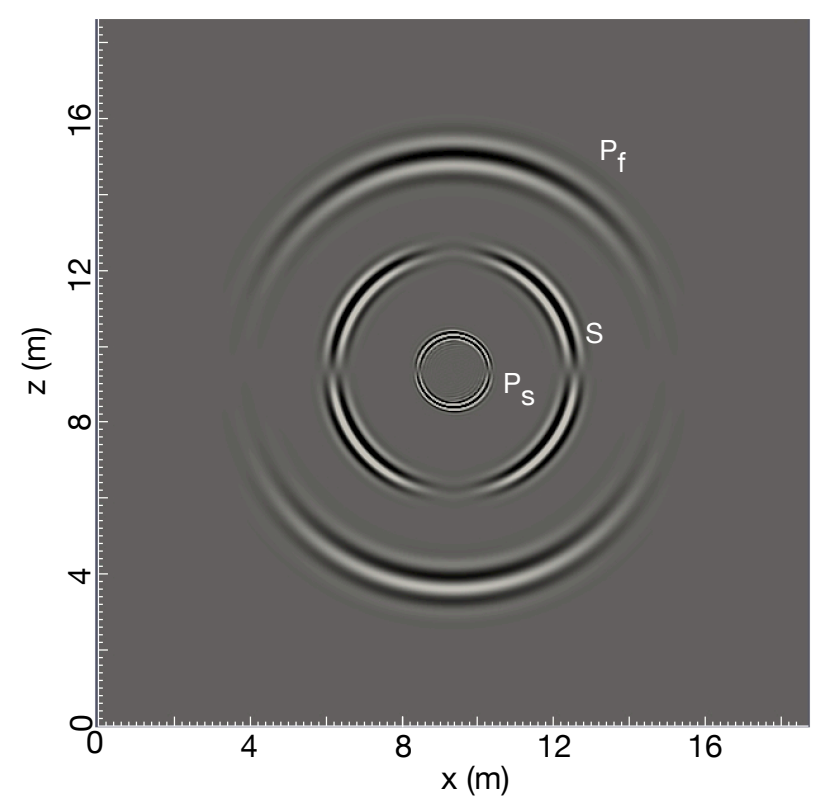

(b) $b_{z}$ at $\eta=0$

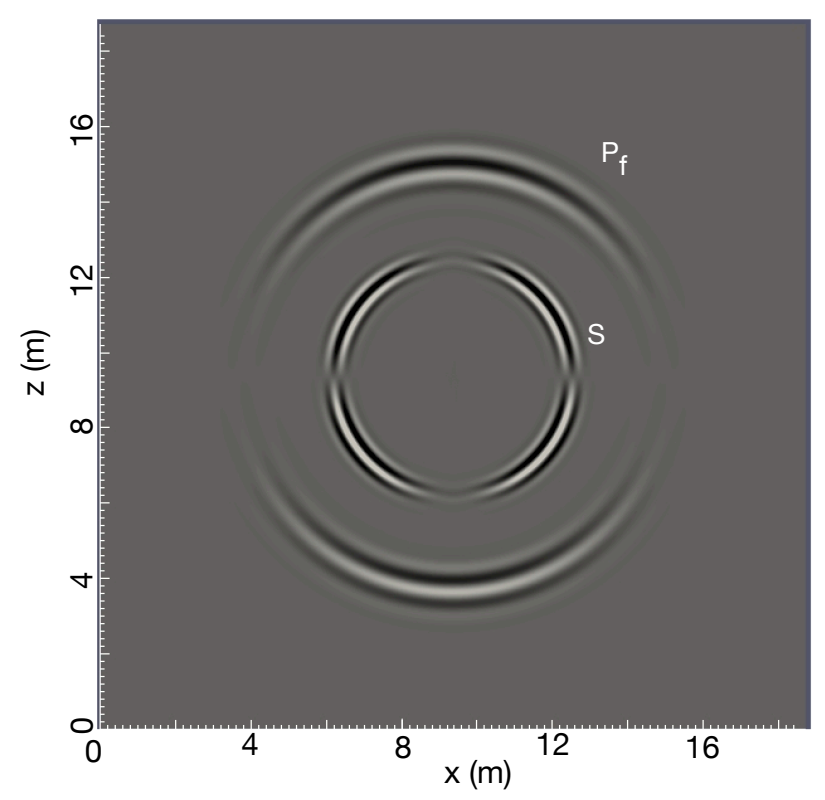

(d) $b_{z}$ at $\eta n e 0$

Fig. 8 Snapshots of the centre of mass particle velocity in isotropic sandstone, computed at $t=2.2 \mathrm{~ms}$, where (a) and (b) corresponds to $\eta=0$, and (c) and (d) corresponds to $\eta \neq 0$. The central frequency of the forcing function is $3730 \mathrm{~Hz}$. The solution is computed for a polynomial of order $4 . \mathrm{P}_{\mathrm{f}}$ : Fast compressional wave, $\mathrm{S}$ : Shear wave, $\mathrm{P}_{\mathrm{s}}$ : slow wave (Biot mode).

the slow $\mathrm{P}$ wave becomes a diffusive mode due to the dominance of viscous forces over the inertial forces.

Figure 5 shows the numerical results for the same poroacoustic medium but with a forcing function of central frequency $\left(f_{c}=4.5 \mathrm{kHz}\right)$. The size of the computational domain in this case is $10 \mathrm{~m} \times 10 \mathrm{~m}$. The minimum size of the mesh is $0.04 \mathrm{~m}$. Figure 5(a) and 5(b) rep- resent the snapshots of the bulk and fluid pressures, respectively, computed at $t=1.2 \mathrm{~ms}$ and $\eta=0$ with a bulk forcing function. Figure 5(c) and 5(d) are the bulk and fluid pressures, respectively, computed for a bulk forcing function at $t=1.2 \mathrm{~ms}$ and $\eta \neq 0$. The physical interpretation of Figure 5 is the same as that of the Figure 4, just at a different scale and the slow 


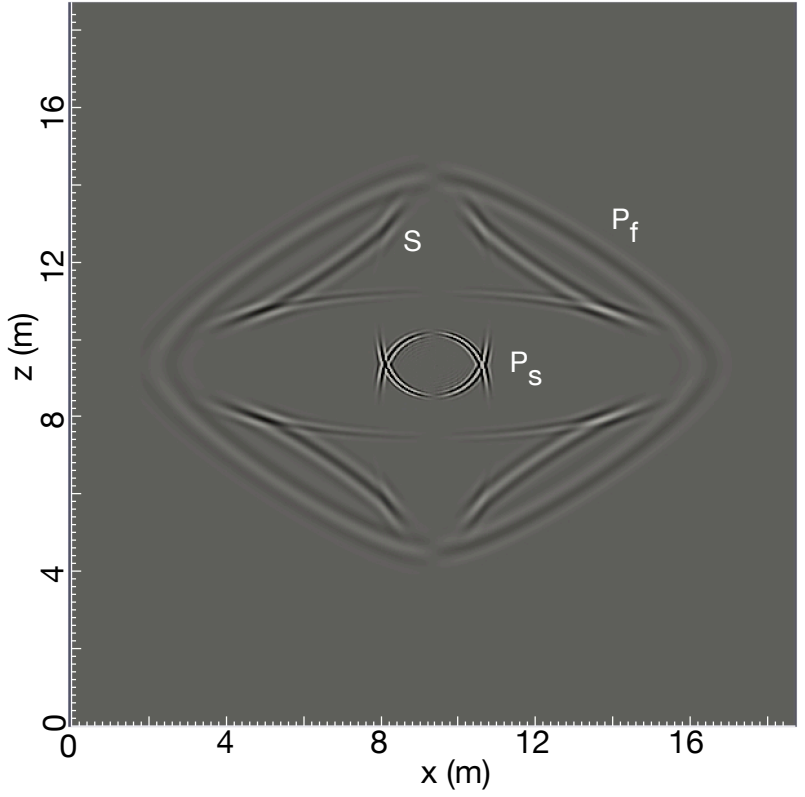

(a) $b_{x}$ at $\eta=0$

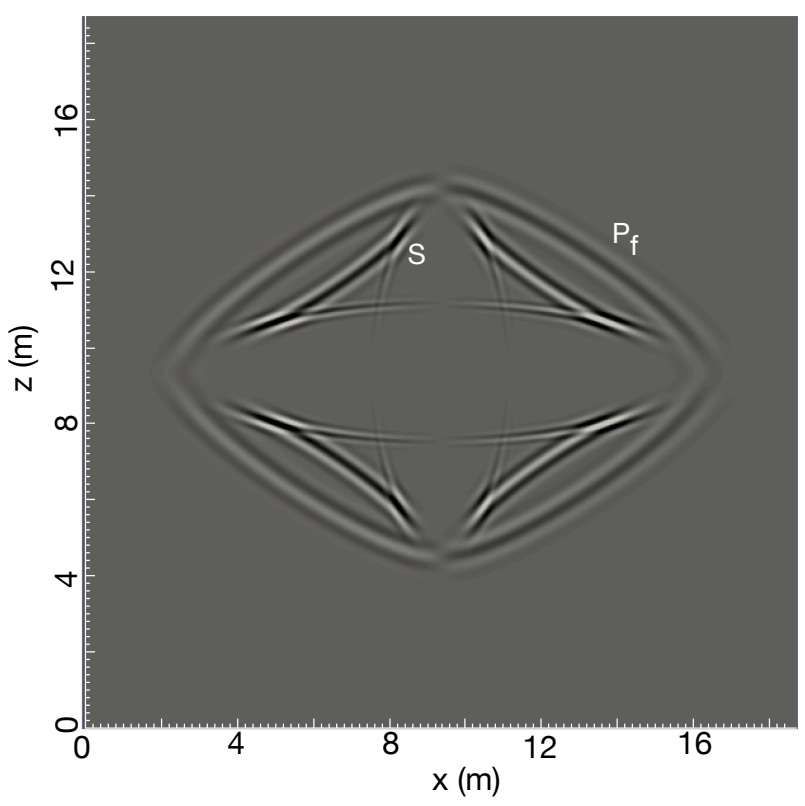

(c) $b_{x}$ at $\eta \neq 0$

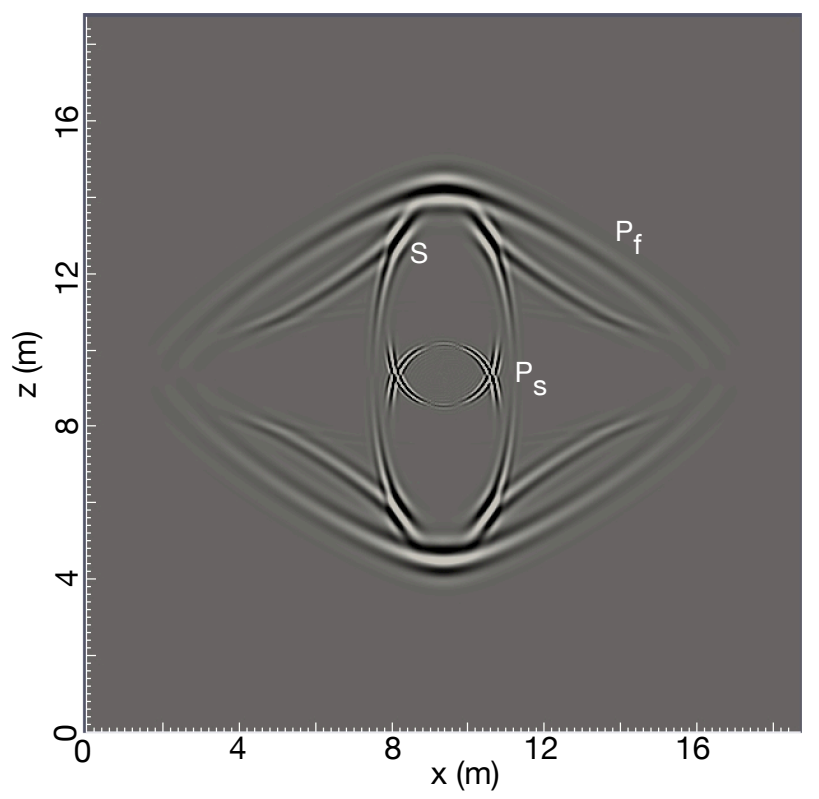

(b) $b_{z}$ at $\eta=0$

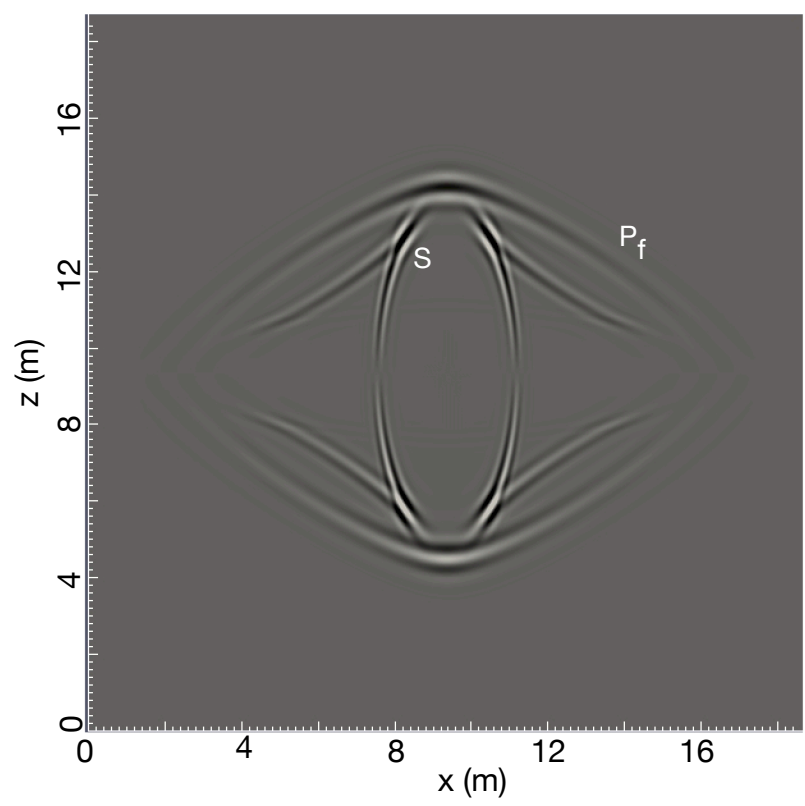

(d) $b_{z}$ at $\eta \neq 0$

Fig. 9 Snapshots of the centre of mass particle velocity in epoxy-glass, computed at $t=1.8 \mathrm{~ms}$, where (a) and (b) corresponds to $\eta=0$, and (c) and (d) corresponds to $\eta \neq 0$. The central frequency of the forcing function is $3730 \mathrm{~Hz}$. The solution is computed for a polynomial of order $4 . \mathrm{P}_{\mathrm{f}}$ : Fast compressional wave, S: Shear wave, $\mathrm{P}_{\mathrm{s}}$ : slow wave (Biot mode).

$\mathrm{P}$ wave propagates faster than those seen in Figure 4. The dispersion analysis also shows a non-zero velocity of the slow $\mathrm{P}$ wave at frequency $4.5 \mathrm{kHz}[8]$.

\subsection{Homogeneous poroelastic medium: Wave-field} simulation

Here we illustrate the effect of anisotropy in (25) using our numerical scheme. We have considered, sandstone (orthotropic and isotropic), epoxy-glass and shale, brine filled, with the material properties given in Table 2. In 


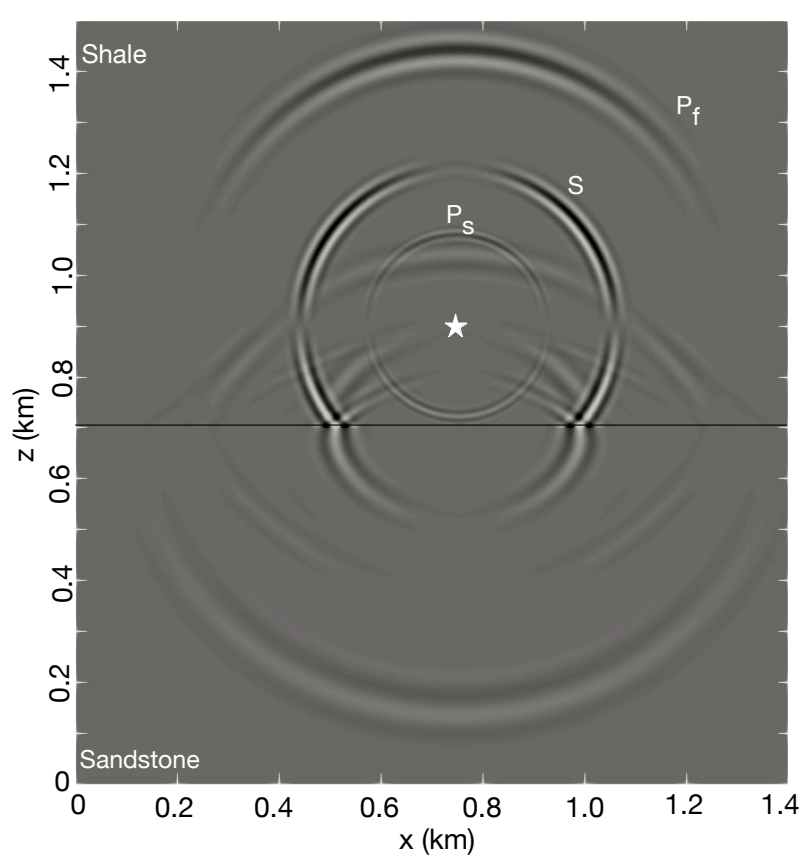

Fig. 10 Snapshot of the $z$-component of the centre of mass particle velocity in an inviscid $(\eta=0)$ heterogenous medium, computed at $t=0.25 \mathrm{~s}$. The central frequency of the forcing function is $45 \mathrm{~Hz}$. The solution is computed for a polynomial of order 4. The star represents the location of the point source perturbation. $\mathrm{P}_{\mathrm{f}}$ : Direct fast compressional wave, $\mathrm{S}$ : Direct shear wave, $\mathrm{P}_{\mathrm{s}}$ : Direct slow wave (Biot mode).

order to have a detailed insight into the results of a poroelastic simulation, the energy velocity surfaces are computed for the materials with properties described in Table 2, solving the eigenvalue problem expressed in (69) in Appendix B. The surfaces include fast compressional, shear, and slow compressional waves with respect to azimuth. Figure $6 \mathrm{a}, 6 \mathrm{~b}, 6 \mathrm{c}$, and $6 \mathrm{~d}$ show the energy velocities of the orthotropic sandstone, isotropic sandstone, epoxy-glass, and shale, respectively. The geometry of the energy velocity surface always agrees with the trajectory of the advancing wavefronts of the modes.

We have carried out numerical simulations with our scheme in order to compare with the energy velocity surfaces. In the subsequent discussions the field represents the center of mass particle velocity vector [28], which is expressed as

$\mathbf{b}=\mathbf{v}+\left(\frac{\rho_{f}}{\rho}\right) \mathbf{q}$

The forcing function, in the subsequent simulation is given in (58) with a nonzero force corresponding to a vertical stress $\sigma_{z z}$ and a fluid pressure $p$. The size of the computational domain is $18.25 \mathrm{~m} \times 18.25 \mathrm{~m}$. The minimum edge length is $5 \mathrm{~cm}$.

Figure 7(a)-(d) represents the $x$ and $z$ components of the center of mass particle velocity of the orthotropic sandstone, where (a) and (b) correspond to the inviscid case $(\eta=0)$, and (c) and (d) to the viscid case $(\eta=0)$. The central frequency of the forcing function is $f_{c}=3730 \mathrm{~Hz}$, and the basis functions have a polynomial degree $N=4$. The propagation time is $1.6 \mathrm{~ms}$. Three events can be clearly observed: the fast $\mathrm{P}$ mode $\left(\mathrm{P}_{\mathrm{f}}\right.$, outer wavefront), the shear wave ( $\mathrm{S}$, middle wavefront), and the slow $\mathrm{P}$ mode $\left(\mathrm{P}_{\mathrm{s}}\right.$, inner wavefront). In the viscid case, the slow mode diffuses faster and the medium behaves almost as a single phase medium.

Figure 8(a)-(d) show the $x-$ and $z$ - components of the center of mass particle velocity in an isotropic sandstone, where (a) and (b) correspond to the inviscid case $(\eta=0)$, and (c) and (d) to the viscid case $(\eta=0)$. Figure 8 is produced with the same simulation parameters as those in Figure 7 except that the propagation time is $2.2 \mathrm{~ms}$. The physical significance of Figure 8 is the same as in Figure 7 except the fact that the radiation pattern is azimuthally invariant. The trajectory of the wavefronts mimics the surfaces of the energy velocity presented in Figure 6(b).

Snapshots of the $x$ - and $z$ - components of the center of mass particle velocity in the epoxy-glass porous medium are represented in Figure 9. Figures 9(a) and (b) correspond to the inviscid case $(\eta=0)$, and (c) and (d) to the viscid case $(\eta=0)$. The central frequency is $f_{c}=3135 \mathrm{~Hz}$. The propagation time is $1.9 \mathrm{~ms}$. It is worth noting the cuspidal triangles of $\mathrm{S}$ and $\mathrm{P}_{\mathrm{S}}$ which is a typical phenomena in anisotropic materials. At $45^{\circ}$, the polarization of the $\mathrm{P}_{\mathrm{s}}$ mode wave is almost horizontal, which confirms the results shown in Figure $3(\mathrm{~b})$ of [9].

\subsection{Heterogenous poroelastic medium: Wave-field simulation}

With this last example, we illustrate the effect of an interface between two porous media. A two-layer model comprising shale and sandstone, both of them filled with brine, is constructed. The size of the computational domain is $1400 \mathrm{~m} \times 1500 \mathrm{~m}$ in the $x$ and $z$ directions, respectively. The minimum size of the edge of the triangular element, used to triangulate the domain, is $8 \mathrm{~m}$. The forcing function is located at $(750 \mathrm{~m}, 900 \mathrm{~m})$ The propagation time is $0.25 \mathrm{~s}$. A Snapshot of the $z$ component of the center of mass particle velocity is represented in Figure 10 for an inviscid case $(\eta=0)$. Figure 11 clearly shows the direct, reflected, and transmitted wavefronts, corresponding to all three modes. The slow $\mathrm{P}$ wave is more prominent in the shale. 


\section{Discussion}

We have developed a nodal DG-method-based approach to simulate poroelastic wave phenomena and demonstrated its ability to generate correct solutions in both homogeneous and heterogeneous domains. In the proposed method, we use the Lax-Friedrich flux, which extends from isotropic to anisotropic media naturally, unlike the upwind flux used in [31]. The poroelastic system has very complex Jacobian matrices, which pose a computational challenge for the eigen-decomposition. The Lax-Freidrich flux is slightly more dissipative than the upwind flux but the effects of numerical dissipation is less prominent at very high order [20,12]. Another challenge in the poroelastic system is to circumvent the effect of the stiffness, caused by strong dissipation at low frequencies. In the present work, we address the stiffness by using a first-order operator splitting approach. This operator deteriorates the convergence rate for a viscid case. We find that it works reasonably well for all spatial orders tested, i.e., $N=1 . .4$. Existing alternatives, include [13] who use a locally implicit time integration scheme. Our scheme is simpler but fully explicit.

\section{Conclusions}

We have proved the well-posedness of the poroelastic system by showing that the energy rate in the system is bounded. We also have proposed a numerical scheme in strong form based on the nodal discontinuous Galerkin finite-element method, paired with a first-order operator splitting approach to handle the stiffness present in the system. We have proved the accuracy of the proposed scheme by comparing the numerical solution with an analytical solution. A convergence study shows $O\left(h^{N+1}\right)$ accuracy. We have further simulated the wave fields for various real-case scenarios comprising homogenous and heterogeneous materials with anisotropy. The simulation correctly produces the fast and slow compressional waves along with the shear waves.

Acknowledgements KS would like to acknowledge the School of Geology, OSU and the MCSS, EPFL Switzerland, for providing the fund to carry out this work. We also acknowledge the OGS, Italy for hosting KS at various occasions.

\section{References}

1. Allard, J., Atalla, N.: Propagation of sound in porous media: modelling sound absorbing materials. John Wiley \& Sons (2009)

2. Badiey, M., Jaya, I., Cheng, A.H.: Propagator matrix for plane wave reflection from inhomogeneous anisotropic poroelastic seafloor. Journal of Computational Acoustics 2, 11-27 (1994)

3. Biot, M.A.: Theory of propagation of elastic waves in a fluid-saturated porous solid: I. Low frequency range. The Journal of the acoustical Society of america, 28, 168-178 (1956)

4. Biot, M.A.: Theory of propagation of elastic waves in a fluid-saturated porous solid: II. Higher frequency range. The Journal of the acoustical Society of america, 28, 179191 (1956)

5. Biot, M.A.: Mechanics of deformation and acoustic propagation in porous media. Journal of applied physics 33, 14821498 (1962).

6. Cockburn, B., Shu, C.W.: Runge-Kutta discontinuous Galerkin methods for convection-dominated problems. Journal of scientific computing 16, 173-261 (2001)

7. Coussy, O., Zinszner, B.: Acoustics of porous media. Editions Technip (1987)

8. Carcione, J.M., Quiroga-Goode, G.: Some aspects of the physics and numerical modeling of Biot compressional waves. Journal of Computational Acoustics 3, 261-280 (1995)

9. Carcione, J.M.: Wave propagation in anisotropic, saturated porous media: Plane-wave theory and numerical simulation. The Journal of the Acoustical Society of America 99, 2655-2666 (1996)

10. Carcione, J.M., Morency, C., Santos, J.E.: Computational poroelasticity-A review. Geophysics 75, 229-243 (2010)

11. Carcione, J.M.: Wave Fields in Real Media: Theory and numerical simulation of wave propagation in anisotropic, anelastic, porous and electromagnetic Media (3rd edition). Elsevier Science (2014)

12. de la Puente, J., Käser, M., Dumbser, M., Igel, H.: An arbitrary high-order discontinuous Galerkin method for elastic waves on unstructured meshes-IV. Anisotropy. Geophysical Journal International 169, 1210-1228 (2007)

13. de la Puente, J., Dumbser, M., Käser, M., Igel, H., 2008. Discontinuous Galerkin methods for wave propagation in poroelastic media. Geophysics 73, 77-97 (2008)

14. Dablain, M.A.: The application of high-order differencing to the scalar wave equation. Geophysics 51, 54-66 (1986)

15. Dai, N., Vafidis, A., Kanasewich, E.R.: Wave propagation in heterogeneous, porous media: A velocity-stress, finitedifference method. Geophysics 60, 327-340 (1995)

16. Dumbser, M., Enaux, C., Toro, E.F.: Finite volume schemes of very high order of accuracy for stiff hyperbolic balance laws. Journal of Computational Physics 227, 39714001 (2008)

17. Etgen, J.T., Dellinger, J.: Accurate wave-equation modeling. SEG Technical Program Expanded Abstracts, 494-497 (1989)

18. Garg, S.K., Nayfeh, A.H., Good, A.J.: Compressional waves in fluid-saturated elastic porous media. Journal of Applied Physics 45, 1968-1974 (1974)

19. Grote, M.J., Schneebeli, A., Schötzau, D., 2006. Discontinuous Galerkin finite element method for the wave equation. SIAM Journal on Numerical Analysis 44, 2408-2431 (2006)

20. Hesthaven, J.S., Warburton, T.: Nodal discontinuous Galerkin methods: algorithms, analysis, and applications. Springer Science \& Business Media (2007)

21. Hunter, R.J.: Foundations of colloid science. Oxford university press (2001)

22. Käser, M., Dumbser, M., De La Puente, J., Igel, H.: An arbitrary high-order discontinuous Galerkin method for 
elastic waves on unstructured meshes-III. Viscoelastic attenuation. Geophysical Journal International 168, 224-242 (2007)

23. Komatitsch, D., Vilotte, J.P.: The spectral element method: an efficient tool to simulate the seismic response of $2 \mathrm{D}$ and $3 \mathrm{D}$ geological structures. Bulletin of the seismological society of America 88, 368-392 (1998)

24. Lemoine, G.I., Ou, M.Y., LeVeque, R.J.: High-resolution finite volume modeling of wave propagation in orthotropic poroelastic media. SIAM Journal on Scientific Computing 35, 176-206 (2013)

25. Levander, A.R.: Fourth-order finite-difference P-SV seismograms. Geophysics 53, 1425-1436 (1988)

26. LeVeque, R.J.: Finite volume methods for hyperbolic problems. Cambridge university press (2002)

27. Özdenvar, T., McMechan, G.A.: Algorithms for staggered-grid computations for poroelastic, elastic, acoustic, and scalar wave equations. Geophysical Prospecting 45, 403-420 (1997)

28. Sahay, P.N.: Natural field variables in dynamic poroelasticity. SEG Technical Program Expanded Abstracts, Society of Exploration Geophysicists, 1163-1166 (1994)

29. Santos, J.E., Oreña, E.J.: Elastic wave propagation in fluid-saturated porous media- Part II The Galerkin procedures. Mathematical Modelling and Numerical Analysis 20, 129-139 (1986)

30. Virieux, J.: P-SV wave propagation in heterogeneous media: Velocity-stress finite-difference method. Geophysics 51, 889-901 (1986)

31. Ward, N.D., Lähivaara, T., Eveson, S., 2017. A discontinuous Galerkin method for poroelastic wave propagation: The two-dimensional case. Journal of Computational Physics 350, 690-727 (2017)

32. Ye, R., de Hoop, M.V., Petrovitch, C.L., Pyrak-Nolte, L.J., Wilcox, L.C.: A discontinuous Galerkin method with a modified penalty flux for the propagation and scattering of acousto-elastic waves. Geophysical Journal International 205, 1267-1289 (2016)

\section{Appendix}

\section{A Solution of the stiff part}

The system of equations represented by (47) is expressed as

$\partial_{t} v_{x}=-\frac{\eta}{\kappa_{1}} \beta_{12}^{(1)} q_{x}$

$\partial_{t} v_{z}=-\frac{\eta}{\kappa_{3}} \beta_{12}^{(3)} q_{z}$

$\partial_{t} q_{x}=-\frac{\eta}{\kappa_{1}} \beta_{22}^{(1)} q_{x}$

$\partial_{t} q_{z}=-\frac{\eta}{\kappa_{3}} \beta_{22}^{(3)} q_{z}$

The solution of equations (60)-(63) is given as

$v_{x}=v_{x}^{n}+\frac{\beta_{12}^{(1)}}{\beta_{22}^{(1)}}\left[\exp \left(-\frac{\eta}{\kappa_{1}} \beta_{22}^{(1)} d t\right)-1\right] q_{x}^{n}$,

$v_{z}=v_{z}^{n}+\frac{\beta_{12}^{(3)}}{\beta_{22}^{(3)}}\left[\exp \left(-\frac{\eta}{\kappa_{3}} \beta_{22}^{(3)} d t\right)-1\right] q_{z}^{n}$,

$q_{x}=\exp \left(-\frac{\eta}{\kappa_{1}} \beta_{22}^{(1)} d t\right) q_{x}^{n}$,

$q_{z}=\exp \left(-\frac{\eta}{\kappa_{3}} \beta_{22}^{(3)} d t\right) q_{z}^{n}$.

\section{B Computation of $\lambda$ in (56)}

A plane-wave solution for the particle velocity vector $V=$ $\left[v_{x}, v_{z}, q_{z}, q_{z}\right]^{T}$ is

$\mathbf{V}=\mathbf{V}_{\mathbf{o}} \exp [\mathrm{i}(\mathbf{k} \cdot \mathbf{x}-\omega t)]$

where $\mathbf{V}_{\mathbf{0}}$ is a constant complex vector and $\mathbf{k}$ is wave vector. Substituting (68) in (1)-(4) and (20)-(23), we recover

$\left(\boldsymbol{\Gamma}^{-1} \cdot \mathbf{L} \cdot \mathbf{C}-V \mathbf{I}_{4}\right) \cdot \mathbf{V}=0$,

where

$\boldsymbol{\Gamma}=\left[\begin{array}{cccc}\rho & 0 & \rho_{f} & 0 \\ 0 & \rho & 0 & \rho_{f} \\ \rho_{f} & 0 & \mathrm{i} Y_{1}(-\omega) / \omega & 0 \\ 0 & \rho_{f} & 0 & \mathrm{i} Y_{3}(-\omega) / \omega\end{array}\right], \quad \mathbf{L}=\left[\begin{array}{cccc}l_{x} & 0 & l_{z} & 0 \\ 0 & l_{z} & l_{x} & 0 \\ 0 & 0 & 0 & l_{x} \\ 0 & 0 & 0 & l_{z}\end{array}\right]$

$\mathbf{C}=\left[\begin{array}{cccc}l_{x} c_{11}^{u} & l_{z} c_{13}^{u} & \alpha_{1} M l_{x} & \alpha_{1} M l_{z} \\ l_{x} c_{13}^{u} & l_{z} c_{33}^{u} & \alpha_{3} M l_{x} & \alpha_{3} M l_{z} \\ l_{z} c_{55}^{u} & l_{x} c_{55}^{u} & 0 & 0 \\ \alpha_{1} M l_{x} & \alpha_{3} M l_{z} & M l_{x} & M l_{z}\end{array}\right]$

with $Y_{i}(\omega)=\mathrm{i} \omega m_{i}+\eta / \kappa_{i}$ and $l_{x}$ and $l_{z}$ being direction cosines and $V=\frac{\omega^{2}}{k^{2}}$.

Term $\mathbf{V}$ in (69) represents the phase velocity of waves and can be computed by adopting the approach for eigenvalue computation. Thus

$\lambda_{i}=\left(\operatorname{Re}\left(1 / V_{i}\right)\right)$ for $i=1 \ldots 4$,

and $\lambda=\max \left(\lambda_{i}\right)$

Energy velocity $\mathbf{V}_{\mathbf{e}}$ can be computed from

$\mathbf{k}^{\mathbf{T}} \cdot \mathbf{V}_{\mathbf{e}}=\mathbf{V}$

\section{System of poroacoustic wave equation}

This system is

$\partial_{t} \mathbf{q}_{\mathbf{p}}+\mathbf{A}_{\mathbf{1} \mathbf{p}} \partial_{x} \mathbf{q}_{\mathbf{p}}+\mathbf{B}_{\mathbf{1} \mathbf{p}} \partial_{x} \mathbf{q}_{\mathbf{p}}=\mathbf{D}_{\mathbf{1} \mathbf{p}} \mathbf{q}_{\mathbf{p}}+\mathbf{f}_{\mathbf{p}}$

where

$\mathbf{q}_{\mathbf{p}}=\left[\begin{array}{llllll}v_{x} & v_{z} & q_{x} & q_{z} & p & p_{f}\end{array}\right]^{T}$,

with $p$ being the bulk pressure, $p_{f}$ is fluid pressure, $v^{\prime s}$ and $q^{\prime s}$ are solid and fluid particle velocity (relative to solid). $\mathbf{A}_{\mathbf{1}} \mathbf{p}$ 
$\mathbf{B}_{\mathbf{1 p}}$ and $\mathbf{D}_{\mathbf{1 p}}$ are defined as

$$
\begin{aligned}
\mathbf{A}_{1 \mathbf{p}}=- & {\left[\begin{array}{cccccc}
0 & 0 & 0 & 0 & \beta_{11} & \beta_{12} \\
0 & 0 & 0 & 0 & 0 & 0 \\
0 & 0 & 0 & 0 & -\beta_{21} & -\beta_{22} \\
0 & 0 & 0 & 0 & 0 & 0 \\
-H & 0 & -C & 0 & 0 & 0 \\
-C & 0 & -M & 0 & 0 & 0
\end{array}\right], } \\
\mathbf{B}_{1 \mathbf{p}}= & -\left[\begin{array}{cccccc}
0 & 0 & 0 & 0 & 0 & 0 \\
0 & 0 & 0 & 0 & \beta_{11} & \beta_{12} \\
0 & 0 & 0 & 0 & 0 & 0 \\
0 & 0 & 0 & 0 & -\beta_{21} & -\beta_{22} \\
0 & -H & 0 & -C & 0 & 0 \\
0 & -C & 0 & -M & 0 & 0
\end{array}\right], \\
\mathbf{D}_{1 \mathbf{p}}=- & {\left[\begin{array}{cccccc}
0 & 0 & \frac{\eta}{\kappa} \beta_{12} & 0 & 0 & 0 \\
0 & 0 & 0 & \frac{\eta}{\kappa} \beta_{12} & 0 & 0 \\
0 & 0 & -\frac{\eta}{\kappa} \beta_{22} & 0 & 0 & 0 \\
0 & 0 & 0 & -\frac{\eta}{\kappa} \beta_{22} & 0 & 0 \\
0 & 0 & 0 & 0 & 0 & 0 \\
0 & 0 & 0 & 0 & 0 & 0
\end{array}\right], }
\end{aligned}
$$

where $\beta$ 's, $H, C$ and $M$ are dependent on the solid bulk modulus $\left(K_{s}\right)$, the fluid bulk modulus $\left(K_{f}\right)$, the solid density $\left(\rho_{s}\right)$, the porosity $(\phi)$, the permeability $(\kappa)$, the fluid density $\left(\rho_{f}\right)$ and the viscosity $(\eta)$ of the medium, elaborately expressed in [8]. 